\title{
Korn Inequalities for a Reinforced Plate
}

\author{
Sergey A. Nazarov • Andrey S. Slutskij • \\ Guido H. Sweers
}

Received: 2 September 2008 / Published online: 4 December 2010

(C) Springer Science+Business Media B.V. 2010

\begin{abstract}
Asymptotically optimal Korn inequalities are derived for a composite material that consists of two families of stiff rods surrounded by a homogeneous soft material. The composite plate is fixed through the protruding stiff rods only. The asymptotic behaviour is shown to be crucially different for families of connected rods and for those where the rods are isolated.
\end{abstract}

Keywords Korn inequality $\cdot$ Homogenisation $\cdot$ Reinforced plate

Mathematics Subject Classification (2000) $74 \mathrm{~K} 30 \cdot 35 \mathrm{Q} 72 \cdot 74 \mathrm{~K} 10$

\section{Introduction}

\subsection{Definition of a Reinforced Plate}

We consider a composite material that consists of a perpendicular mesh of two families of stiff fibres surrounded by a matrix of homogeneous softer material. These stiff fibres pro-

The authors gratefully acknowledge support by N.W.O., the Netherlands Organisation for Scientific Research.

S.A. Nazarov · A.S. Slutskij

Institute of Mechanical Engineering Problems, V.O., Bol'shoi pr., 61, 199178 St.-Petersburg, Russia

S.A. Nazarov

e-mail: serna@snark.ipme.ru

e-mail:srgnazarov@yahoo.co.uk

A.S. Slutskij

e-mail: slutskij@gmail.com

G.H. Sweers $(\bowtie)$

Mathematisches Institut der Universität zu Köln, Weyertal 86-90, 50931 Köln, Germany

e-mail: gsweers@math.uni-koeln.de

G.H. Sweers

DIAM-EWI, Delft University of Technology, P.O. Box 5031, 2600GA Delft, Netherlands 


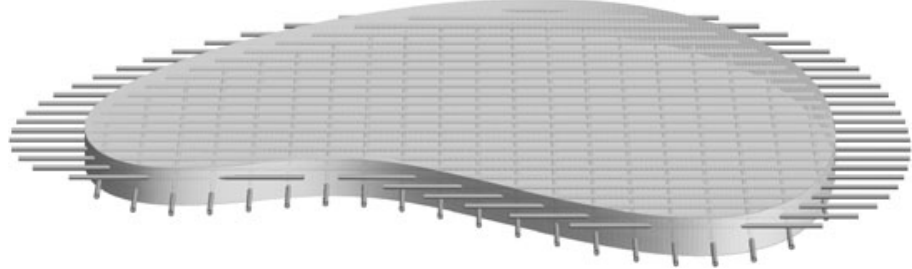

Fig. 1 A sketch of the composite material containing two families of fibers in a matrix of another material. The matrix of which the band is the lateral surface is supposed to consist of softer material
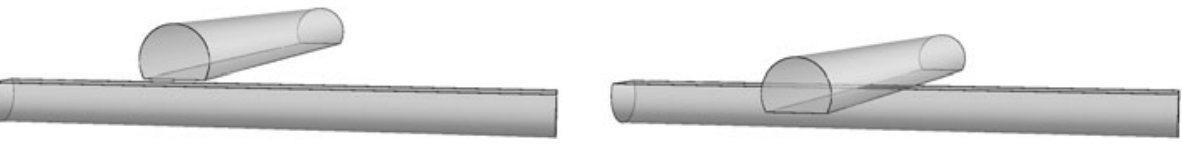

Fig. 2 Isolated rods and welded rods
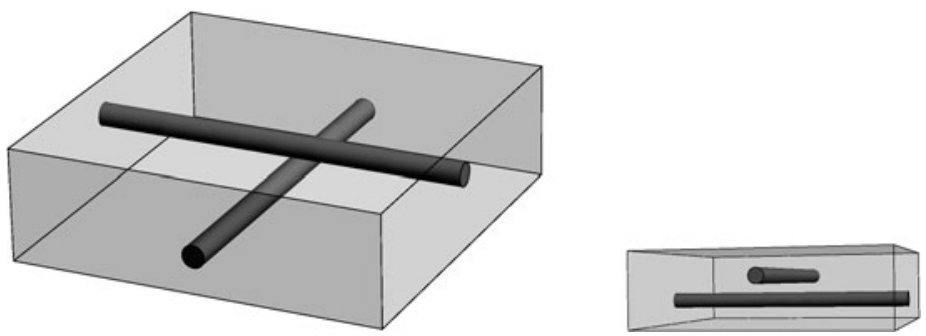

Fig. 3 A periodicity cell with disjoint rods
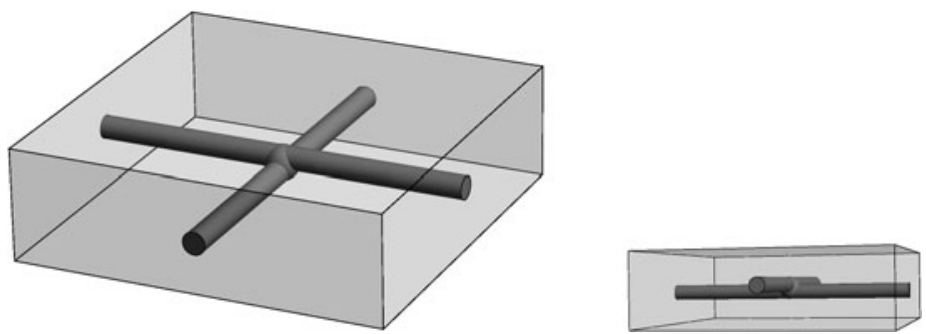

Fig. 4 A periodicity cell with welded rods

trude from the soft elastic material and are fixed at their ends. See Fig. 1. We will compare the asymptotic behaviour for two different cases, namely, 1) the case that the two families of fibers are only connected through the surrounding softer material, and 2) the fibers themselves are connected whenever they cross. This last situation can be considered as the two families of rods being welded. See Fig. 2. Away from the sides the material is periodic in both horizontal directions. For a typical periodicity cell see Figs. 3 and 4.

On first sight both situations might seem quite similar. A closer observation however will show that the asymptotic behaviour in these two situations will differ crucially. The most striking difference is that the limit problem in the first case is no longer elliptic. 
The precise setting is as follows.

- The parameter $h \ll 1$ will denote the thickness of the plate. Moreover $h$ will also be the distance of two consecutive rods and we also fix the diameter of the rods as $\mathcal{O}(h)$.

- The open cross-sections of the unscaled rods we name $\pi_{1}, \pi_{2} \subset \mathbb{R}^{2}$ with $\overline{\pi_{1}} \subset\left(-\frac{1}{2}, \frac{1}{2}\right) \times$ $\left[0, \frac{1}{2}\right)$, and $\overline{\pi_{2}} \subset\left(-\frac{1}{2}, \frac{1}{2}\right) \times\left(-\frac{1}{2}, 0\right]$. We assume that the two sets of rods will not intersect but might have a common face (a 'welded' connection) at level $z=0$.

We define for each $j \in \mathbb{Z}$ the infinite rods

$$
\begin{aligned}
& \Pi_{h}^{1, j}:=\left\{\left(y_{1}, y_{2}, z\right) \in \mathbb{R}^{3} ; h^{-1}\left(y_{2}-j, z\right) \in \pi_{1}\right\}, \\
& \Pi_{h}^{2, j}:=\left\{\left(y_{1}, y_{2}, z\right) \in \mathbb{R}^{3} ; h^{-1}\left(y_{1}-j, z\right) \in \pi_{2}\right\} .
\end{aligned}
$$

In principle these rods could also 'intersect'; the result will be identical except for the constants. It would slightly complicate the model. We do not consider rods that touch in a set with Hausdorff dimension less than 2.

- For the entire plate we take the cylinder

$$
\Omega_{h}:=\omega \times\left(-\frac{1}{2} h, \frac{1}{2} h\right),
$$

where $\omega \subset \mathbb{R}^{2}$ is a bounded Lipschitz domain. We will also need the $h$-neighborhood of $\omega$ :

$$
\omega(h):=\left\{y \in \mathbb{R}^{2} ; \operatorname{dist}(y, \omega)<h\right\} .
$$

- The rods are supposed to be fixed outside of $\omega(h) \times\left(-\frac{1}{2} h, \frac{1}{2} h\right)$, that is, we consider

$$
\Pi^{i, j}(h):=\Pi_{h}^{i, j} \cap\left(\omega(h) \times\left(-\frac{1}{2} h, \frac{1}{2} h\right)\right) .
$$

For the end of the rods we write

$$
\Gamma^{i, j}(h):=\Pi_{h}^{i, j} \cap\left(\partial \omega(h) \times\left(-\frac{1}{2} h, \frac{1}{2} h\right)\right) .
$$

So the volume that each family of rods fills up is

$$
\Pi^{i}(h):=\bigcup_{j \in \mathbb{Z}} \Pi^{i, j}(h) \quad \text { with } i=1,2 .
$$

We will consider the junction to be fixed at the set

$$
\Gamma^{i}(h):=\bigcup_{j \in \mathbb{Z}} \Gamma^{i, j}(h) \quad \text { with } i=1,2 .
$$

The number of rods that each family $\Pi^{i}(h)$ contains is of order $h^{-1}$.

- The matrix of the soft composite is but the set

$$
\Theta(h)=\Omega_{h} \backslash\left(\overline{\Pi^{1}(h)} \cup \overline{\Pi^{2}(h)}\right) .
$$

The full junction that we study is now described by

$$
\Xi(h)=\Theta(h) \cup \Pi^{1}(h) \cup \Pi^{2}(h) .
$$




\subsection{The Elastic Energy Functional}

We use a matrix notation in defining the relations of the linear elasticity theory (see e.g., [17, 21]). The displacement vector is interpreted as the column $u=\left(u_{1}, u_{2}, u_{3}\right)^{\top}$, where $\top$ denotes the transposition. Let $D\left(\nabla_{x}\right)$ be the following $(6 \times 3)$-matrix of first-order differential operators:

$$
D\left(\nabla_{x}\right)^{\top}=\left(\begin{array}{cccccc}
\partial_{1} & 0 & 0 & 0 & \frac{1}{\sqrt{2}} \partial_{3} & \frac{1}{\sqrt{2}} \partial_{2} \\
0 & \partial_{2} & 0 & \frac{1}{\sqrt{2}} \partial_{3} & 0 & \frac{1}{\sqrt{2}} \partial_{1} \\
0 & 0 & \partial_{3} & \frac{1}{\sqrt{2}} \partial_{2} & \frac{1}{\sqrt{2}} \partial_{1} & 0
\end{array}\right),
$$

where $\nabla_{x}=\left(\partial_{1}, \partial_{2}, \partial_{3}\right)^{\top}$ and $\partial_{j}=\partial / \partial x_{j}$. Then, using the definition of the cartesian components of the strain tensor

$$
\varepsilon_{m n}(u)=\frac{1}{2}\left(\frac{\partial u_{n}}{\partial x_{m}}+\frac{\partial u_{m}}{\partial x_{n}}\right), \quad m, n \in\{1,2,3\},
$$

we obtain the formula

$$
\varepsilon(u)=D\left(\nabla_{x}\right) u
$$

for the strain column

$$
\varepsilon(u)=\left(\varepsilon_{11}(u), \varepsilon_{22}(u), \varepsilon_{33}(u), \sqrt{2} \varepsilon_{23}(u), \sqrt{2} \varepsilon_{13}(u), \sqrt{2} \varepsilon_{23}(u)\right)^{\top} .
$$

The factors $\sqrt{2}$ are introduced in order to have equal norms for the tensor and the columns representing them.

For any bounded domain $\Lambda \in \mathbb{R}^{3}$, we consider the functional

$$
\mathcal{E}(u ; \Lambda)=\sum_{m, n=1}^{3} \int_{\Lambda}\left|\varepsilon_{m n}(u)\right|^{2} d x=\left\|\varepsilon(u) ; L_{2}(\Lambda)\right\|^{2}
$$

as a replacement of the elastic energy functional. For a standard elastic material the elastic energy functional contains, next to strain terms, terms depending on the stress. With the linear relation between stress and strain in the present situation such an energy functional is equivalent to (1.3).

The column $\varepsilon(u)$ and the functional $\mathcal{E}(u ; \Lambda)$ vanish on the linear space $\mathbf{R}$ of rigid motions

$$
\mathbf{R}=\left\{u: \mathbb{R}^{3} \rightarrow \mathbb{R}^{3} ; u(x)=d(x) b, b \in \mathbb{R}^{6}\right\},
$$

where $d(x)$ is the $(6 \times 3)$-matrix

$$
d(x)=\left(\begin{array}{cccccc}
1 & 0 & 0 & 0 & \frac{1}{\sqrt{2}} x_{3} & -\frac{1}{\sqrt{2}} x_{2} \\
0 & 1 & 0 & -\frac{1}{\sqrt{2}} x_{3} & 0 & \frac{1}{\sqrt{2}} x_{1} \\
0 & 0 & 1 & \frac{1}{\sqrt{2}} x_{2} & -\frac{1}{\sqrt{2}} x_{1} & 0
\end{array}\right) .
$$

It this paper we consider the functional

$$
\mathcal{E}_{\gamma}(u ; \Xi(h))=\sum_{i=1,2} \mathcal{E}\left(u ; \Pi^{i}(h)\right)+\gamma \mathcal{E}(u ; \Theta(h)),
$$


where $\gamma>0$ is a parameter characterizing the relative rigidity of the matrix $\Theta(h)$ compared with the fibers $\Pi^{i}(h)$. The case $\gamma \ll 1$ corresponds to soft fibers while $\gamma \gg 1$ represents hard ones. We obtain weighted Korn inequalities for the junction $\Xi(h)$ with weight factors that depend on the geometrical parameter $h$ and the physical parameter $\gamma$. The constants themselves in these inequalities will be independent of $h$ and $\gamma$.

\subsection{Anisotropic and Weighted Korn Inequalities}

Known approaches to prove the Korn inequality

$$
\left\|\nabla_{x} u ; L_{2}(\Omega)\right\|^{2} \leq k(\Omega)\left(\mathcal{E}(u ; \Omega)+\left\|\nabla_{x} u ; L_{2}(B)\right\|^{2}\right),
$$

where $B$ is a subdomain of $\Omega$ (cf. [6, 7, 15, 16, 26, 27] and others), cannot directly describe the dependence of the Korn constant $k(\Omega)$ on the specific geometry of the domain $\Omega$. For domains $\Omega$ and $B$, which are star-shaped with respect to all points in a ball $B=B_{r}$ of radius $r>0$, it was discovered in [13] (see also [14]) that

$$
k(\Omega) \leq C\left(r^{-1} \operatorname{diam} \Omega\right)^{3},
$$

where $C$ is an absolute constant. However, for a thin domain this result is not optimal, since $r=O(h)$ and diam $\Omega_{h}=O(1)$ while $k\left(\Omega_{h}\right), B_{r}$ is known to be $O\left(h^{-2}\right)$, not $O\left(h^{-3}\right)$ as would follow from (1.7) and (1.6).

To the authors' best knowledge, it was in [28] where the concept of an anisotropic Korn inequality was introduced in order to justify the Kirchhoff's plate theory, namely, the Sobolev norm of $u$ in $H^{1}(\Omega)$ has to be supplied with weight factors that differ for transversal and longitudinal displacements, and their derivatives in those directions (see (2.3) with $\rho_{h}=1$ ). Then, in [18] (see also [21, Chap. 3], [23, Sect. 2]) an additional weight of type (2.2) was put into the Sobolev norm. This weight uses the distance of a point $x$ to the clamped part of the plate. Such a weight factor becomes a crucial tool in the derivation of an asymptotically sharp (with respect to the small parameter) form of Korn's inequality for junctions of thin plates (see [11]). Concerning junctions of massive bodies and thin rods we refer to $[2-5,8-10,12,19,24]$ and the review [23]. We emphasize that the final form of an asymptotically sharp weighted anisotropic Korn inequality is always very sensitive to the global geometry of a junction including the type of boundary conditions.

In the present paper we provide a weighted anisotropic norm $|u|_{\Xi(h)}$ for $u$ in $H^{1}(\Xi(h))$ such that the constant $c$ in the inequality

$$
|u|_{\Xi(h)}^{2} \leq c \mathcal{E}(u ; \Xi(h))
$$

is independent of both the parameters $h \in(0,1)$ and $\gamma \in(0,+\infty)$. To do so, we efficiently employ several techniques such as weighted anisotropic Korn inequalities for individual rods and the plate, a decomposition of the displacement field into the sum of two components, i.e. a rigid motion $u^{0} \in \mathbf{R}$ and an "orthogonal" component $u^{\perp}$, processed by different tools, the relation (1.6) for tiny parts (the rod and plate periodicity cells), and then coupling the individual Korn inequalities and passing them from one composite fragment to another. Those techniques, developed and used in [12, 18, 22, 24] and others, are described and explained in review [23]. The typical scheme in the paper to improve step by step the quality 
of Korn's inequality, i.e., to enlarge the weights on certain components, becomes

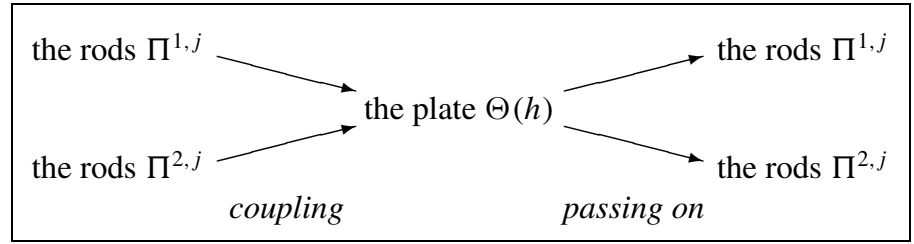

\subsection{Structure of the Paper}

In Sect. 4 we formulate anisotropic weighted Korn inequalities for isolated rods and for a plate, which are known to be asymptotically sharp (see [18, 28] and [23]). In Sect. 3 we formulate the final result for the elastic junction and explain how it differs with the Korn inequality for a junction where the family of rods are welded together (see the periodicity cells in Figs. 3 and 4). We finish the section by sketching some generalizations of our approach.

The proofs are presented in Sects. 5 and 6. We consistently process all the different cases of the relations between the stiffnesses of the rods and the plate which will realize the steps in (1.8). Each of these relations is supplied with an example of a particular displacement field that will demonstrate that the weights we have derived are indeed optimal (see Sects. 5.2, 5.3, 5.4 and 6.2).

\section{The Korn Inequalities for Isolated Components}

\subsection{The Homogeneous Plate}

We start by considering the vector field $u$ defined on the set $\Omega_{h}$ and supplied with the condition

$$
u(x)=0, \quad x \in \Upsilon_{h},
$$

where

$$
\Upsilon_{h}=\left\{(y, z)=\left(y_{1}, y_{2}, z\right): y \in \partial \omega, z \in(-h / 2, h / 2)\right\}
$$

is the lateral surface of $\Omega_{h}$. The condition (2.1) means that the lateral surface $\Upsilon_{h}$ of the plate $\Omega_{h}$ is fixed. Korn inequalities for fixed plates were proved by B.A. Shoikhet [28]. In [18] (see also [21, Chap. 3], [23, Sect. 2]) these inequalities are improved by introducing the weight factor

$$
\rho_{h}(y)=\min \{1, h+\operatorname{dist}(y, \partial \omega)\} \leq 1 .
$$

This weight factor is of order $h$ in the vicinity of the fixed lateral side $\Upsilon_{h}$ of the plate $\Omega_{h}$ and of order $1=h^{0}$ further away from $\Upsilon_{h}$ (see Fig. 5).

In order to formulate the resulting inequality we introduce the weighted norm $\left|\cdot ; \Omega_{h}\right|_{0}$ in the space $H^{1}\left(\Omega_{h}\right)^{3}$ (cf. [18], [21, Chap. 3], [23, Sect. 2]): 


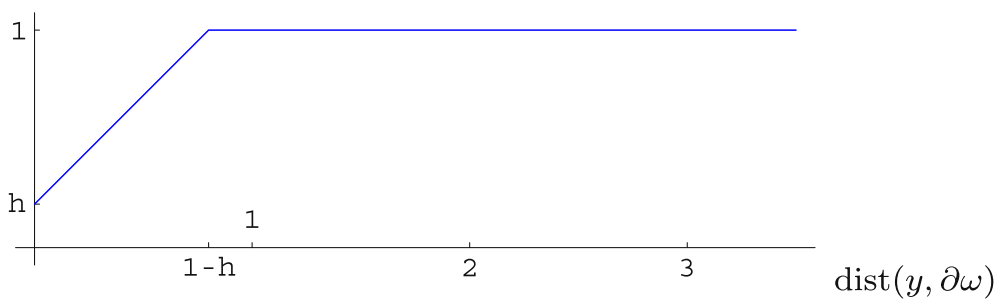

Fig. 5 A graph for the weight factor $y \mapsto \rho_{h}(y)$ as a function of $\operatorname{dist}(y, \partial \omega)$

$$
\begin{aligned}
\left|u ; \Omega_{h}\right|_{0}:= & \left(\int _ { \Omega _ { h } } \left\{\sum_{j=1}^{2}\left[\left|\frac{\partial u_{j}}{\partial y_{1}}\right|^{2}+\left|\frac{\partial u_{j}}{\partial y_{2}}\right|^{2}+\frac{h^{2}}{\rho_{h}^{2}}\left(\left|\frac{\partial u_{j}}{\partial z}\right|^{2}+\left|\frac{\partial u_{3}}{\partial y_{j}}\right|^{2}\right)+\frac{1}{\rho_{h}^{2}}\left|u_{j}\right|^{2}\right]\right.\right. \\
& \left.\left.+\left|\frac{\partial u_{3}}{\partial z}\right|^{2}+\frac{h^{2}}{\rho_{h}^{4}}\left|u_{3}\right|^{2}\right\} d y d z\right)^{1 / 2} .
\end{aligned}
$$

Theorem 2.1 (See $[5,18,21,23])$ For $u \in H_{0}^{1}\left(\Omega_{h}\right)^{3}$ the following inequality holds

$$
\left|u ; \Omega_{h}\right|_{0}^{2} \leq C \mathcal{E}\left(u ; \Omega_{h}\right),
$$

where $C$ is a constant independent of $u$ and $h \in\left(0, h_{0}\right]$.

It is known (see, e.g., $[18,21,23])$ that the distribution of weights in the Sobolev norm (2.3) is optimal, namely it is not possible to enlarge the order in $h$ and $\rho_{h}$ in any of the weights. Let us comment on this distribution. In the vicinity of the fixed lateral side of the plate, weights on derivatives become of order $1=h^{0}$ and on displacements of order $h^{-2}$. Inside the plate, where $\rho_{h}(y)=O(1)$, the longitudinal displacements $u_{1}, u_{2}$ and their derivatives in longitudinal directions keep weights of order 1 , but the transversal displacements $u_{3}$ and the derivatives $\partial u_{3} / \partial y_{1}, \partial u_{3} / \partial y_{2}$ get weights of order $h^{2}$. The latter means that, with the same order of the energy functional the transversal deformation of the plate can be much larger than its longitudinal deformation. One knows this fact from the everyday practice: it is much easier to bend a thin body than to stretch it.

\subsection{Rods}

Let us now consider the vector field $u$ defined on the rod $\Pi^{i, j}(h)$ and supplied with the condition

$$
u(x)=0, \quad x \in \Gamma(h),
$$

i.e. all ends of the rods $\Pi^{i, j}(h) \subset \Pi^{i}(h)$ are fixed. For this separated fixed $\operatorname{rod} \Pi^{i, j}(h)$, we introduce the weighted Sobolev norm (cf. $[18,20]$ and others)

$$
\begin{aligned}
\left|u ; \Pi^{i, j}(h)\right|_{i}:= & \left(\int _ { \Pi ^ { i , j } ( h ) } \left\{\left|\frac{\partial u_{1}}{\partial y_{1}}\right|^{2}+\left|\frac{\partial u_{2}}{\partial y_{2}}\right|^{2}+\left|\frac{\partial u_{3}}{\partial z}\right|^{2}\right.\right. \\
& +\frac{h^{2}}{\rho_{h}^{2}}\left(\left|\frac{\partial u_{2}}{\partial y_{1}}\right|^{2}+\left|\frac{\partial u_{3}}{\partial y_{1}}\right|^{2}+\left|\frac{\partial u_{1}}{\partial y_{2}}\right|^{2}+\left|\frac{\partial u_{3}}{\partial y_{2}}\right|^{2}+\left|\frac{\partial u_{1}}{\partial z}\right|^{2}+\left|\frac{\partial u_{2}}{\partial z}\right|^{2}\right) \\
& \left.\left.+\frac{1}{\rho_{h}^{2}}\left|u_{i}\right|^{2}+\frac{h^{2}}{\rho_{h}^{4}}\left(\left|u_{3-i}\right|^{2}+\left|u_{3}\right|^{2}\right)\right\} d y d z\right)^{1 / 2}
\end{aligned}
$$


Theorem 2.2 (See $[20,21,23])$ For $u \in H_{0}^{1}\left(\Pi^{i, j}(h)\right)^{3}$ there holds the inequality

$$
\left|u ; \Pi^{i, j}(h)\right|_{i}^{2} \leq C \mathcal{E}\left(u ; \Pi^{i, j}(h)\right),
$$

where $C$ is a constant independent of $u$ and $h \in\left(0, h_{0}\right]$.

The distribution of weight that is known to be optimal, is a bit different than in the norm (2.3) for the isolated plate. Again, due to the behavior of $\rho_{h}$ the factors for the displacements and their derivatives take respectively the orders $h^{-2}$ and $h^{0}$ in the vicinity of the clamped end of a rod. In the middle part of a rod the two transversal displacements get the weight $O\left(h^{2}\right)$ (in analogy with $u_{3}$ in the plate) but the longitudinal displacements the weight $O(1)$ (in analogy with $u_{1}$ and $u_{2}$ in the plate). All derivatives have the same weight $O\left(h^{2}\right)$, except for $\partial u_{p} / \partial x_{p}$ which are directly present in the elastic energy functional (1.3).

Remark 2.1 Rods with the cross-section $\omega(h)$ and with the length independent of the small parameter $h$ are considered in [5, 20, 21, 23]. In general, the lengths that appear in $\Pi^{i, j}(h)$ depend on $h$. However, (2.7) can be derived from the above-mentioned results since we may extend the rod $\Pi^{i, j}(h)$ to the left and to the right up to a fixed length and combine the inequalities for those extended rods in order to obtain the necessary weight.

\subsection{A Plate with Welded Rods}

We consider a construction of a plate with welded rods (see Fig. 3) in the case $\gamma=h$. The Korn inequalities for welded rods in this construction is obtained in [1]:

$$
\left|u ; \Pi^{i}(h)\right|_{\times, i}^{2} \leq C \mathcal{E}_{h}\left(u ; \Omega_{h}\right)
$$

where

$$
\begin{aligned}
\left|u ; \Pi^{i}(h)\right|_{\times, i}:= & \left(\int _ { \Pi ^ { i } ( h ) } \left\{\sum_{j=1}^{2}\left[\left|\frac{\partial u_{j}}{\partial y_{1}}\right|^{2}+\left|\frac{\partial u_{j}}{\partial y_{2}}\right|^{2}+\frac{h^{2}}{\rho_{h}^{2}}\left(\left|\frac{\partial u_{j}}{\partial z}\right|^{2}+\left|\frac{\partial u_{3}}{\partial y_{j}}\right|^{2}\right)+\frac{1}{\rho_{h}^{2}}\left|u_{j}\right|^{2}\right]\right.\right. \\
& \left.\left.+\left|\frac{\partial u_{3}}{\partial z}\right|^{2}+\frac{h^{2}}{\rho_{h}^{4}}\left|u_{3}\right|^{2}\right\} d y d z\right)^{1 / 2}
\end{aligned}
$$

and $C$ is a constant independent of $u$ and $h \in\left(0, h_{0}\right]$. The distribution of the weights on the system of welded rods is just the same as in the isolated cylindrical plate (see Sect. 2.1).

For the matrix that we derive in Sect. 5, the following inequality holds:

$$
|u ; \Theta(h)|_{\times, 0}^{2} \leq C \mathcal{E}_{h}\left(u ; \Omega_{h}\right),
$$

where

$$
\begin{aligned}
|u ; \Theta(h)|_{\times, 0}:= & \left(\int _ { \Theta ( h ) } \left\{\sum _ { j = 1 } ^ { 2 } \left[h\left|\frac{\partial u_{j}}{\partial y_{1}}\right|^{2}+h\left|\frac{\partial u_{j}}{\partial y_{2}}\right|^{2}+\min \left\{h, \frac{h^{2}}{\rho_{h}^{2}}\right\}\left(\left|\frac{\partial u_{j}}{\partial z}\right|^{2}+\left|\frac{\partial u_{3}}{\partial y_{j}}\right|^{2}\right)\right.\right.\right. \\
& \left.\left.\left.+\min \left\{\frac{1}{h}, \frac{1}{\rho_{h}^{2}}\right\}\left|u_{j}\right|^{2}\right]+h\left|\frac{\partial u_{3}}{\partial z}\right|^{2}+\min \left\{\frac{1}{h}, \frac{h^{2}}{\rho_{h}^{4}}\right\}\left|u_{3}\right|^{2}\right\} d y d z\right)^{1 / 2} .
\end{aligned}
$$




\section{The Main Results}

\subsection{The Statement}

In order to formulate the Korn inequality for the matrix $\Theta(h)$ we introduce the norm

$$
\begin{aligned}
|u ; \Theta(h)|_{\gamma, 0} & :=\left(\int _ { \Theta ( h ) } \left\{\beta_{1}(h, \gamma, y)\left(\left|u_{1}\right|^{2}+\left|u_{2}\right|^{2}\right)+h^{2} \beta_{2}(h, \gamma, y)\left|u_{3}\right|^{2}\right.\right. \\
& +\beta_{1}(h, \gamma, y) h^{2}\left(\left|\frac{\partial u_{1}}{\partial z}\right|^{2}+\left|\frac{\partial u_{2}}{\partial z}\right|^{2}+\left|\frac{\partial u_{3}}{\partial y_{1}}\right|^{2}+\left|\frac{\partial u_{3}}{\partial y_{2}}\right|^{2}\right) \\
& \left.\left.+\min \{1, \gamma\}\left(\left|\frac{\partial u_{1}}{\partial y_{1}}\right|^{2}+\left|\frac{\partial u_{2}}{\partial y_{2}}\right|^{2}+\left|\frac{\partial u_{3}}{\partial z}\right|^{2}+\left|\frac{\partial u_{1}}{\partial y_{2}}\right|^{2}+\left|\frac{\partial u_{2}}{\partial y_{1}}\right|^{2}\right)\right\} d y d z\right)^{1 / 2},
\end{aligned}
$$

where

$$
\beta_{1}(h, \gamma, y)=\min \left\{\rho_{h}(y)^{-2}, \gamma h^{-2}\right\}, \quad \beta_{2}(h, \gamma, y)=\min \left\{\rho_{h}(y)^{-4}, \gamma h^{-4}\right\} .
$$

In Sect. 5 we prove the next result:

Theorem 3.1 For $u \in H_{0}^{1}(\Xi(h))^{3}$ the following inequality holds

$$
|u ; \Theta(h)|_{\gamma, 0}^{2} \leq c \mathcal{E}_{\gamma}(u ; \Xi(h)),
$$

where $c$ is a constant independent of $u$ and $h \in\left(0, h_{0}\right]$.

Let us consider the fibers $\Pi^{1}(h)$. We set the norm

$$
\begin{aligned}
\left|u ; \Pi^{i}(h)\right|_{i, \gamma}= & \left(\sum _ { j : \Pi ^ { i , j } ( h ) \in \Pi ^ { i } ( h ) } \int _ { \Pi ^ { i , j } ( h ) } \left\{\left|\frac{\partial u_{1}}{\partial y_{1}}\right|^{2}+\left|\frac{\partial u_{2}}{\partial y_{2}}\right|^{2}+\left|\frac{\partial u_{3}}{\partial z}\right|^{2}\right.\right. \\
& +\left(\frac{h^{2}}{\rho_{h}^{2}}+\min \{1, \gamma\}\right)\left(\left|\frac{\partial u_{1}}{\partial y_{2}}\right|^{2}+\left|\frac{\partial u_{2}}{\partial y_{1}}\right|^{2}\right) \\
& +\frac{h^{2}}{\rho_{h}^{2}}\left(\left|\frac{\partial u_{3}}{\partial y_{1}}\right|^{2}+\left|\frac{\partial u_{3}}{\partial y_{2}}\right|^{2}+\left|\frac{\partial u_{1}}{\partial z}\right|^{2}+\left|\frac{\partial u_{2}}{\partial z}\right|^{2}\right) \\
& \left.\left.+\frac{h^{2}}{\rho_{h}^{4}}\left|u_{3}\right|^{2}+\frac{1}{\rho_{h}^{2}}\left|u_{i}\right|^{2}+\left(\frac{h^{2}}{\rho_{h}^{4}}+\beta_{1}\right)\left|u_{3-i}\right|^{2}\right\} d y d z\right)^{1 / 2}
\end{aligned}
$$

and prove in the Sect. 6 the statement:

Theorem 3.2 For $u \in H_{0}^{1}(\Xi(h))^{3}$ the following inequality holds

$$
\left|u ; \Pi^{i}(h)\right|_{i, \gamma} \leq c \mathcal{E}_{\gamma}(u ; \Xi(h)),
$$

where $c$ is a constant independent of $u$ and $h \in\left(0, h_{0}\right]$. 


\subsection{Discussion}

Korn's inequality is the only tool to justify the asymptotic formulae for elastic fields on junctions and for functionals calculated on these fields such as energy, mean rigid motion, eigenfrequences and so on. At the same time, being asymptotically exact in weights and anisotropy, Korn's inequality helps to predict the asymptotic ansatz for the displacements, strains and stresses in the junction.

Let us analyze the difference in the asymptotic ansatz for the composite plates with the periodicity cells drawn in Figs. 3 and 4, which results from the Korn inequalities (2.8), (3.3), (3.5) above, and the results of [1].

We consider the most interesting case, namely $\gamma=c h$, when the bending rigidity of the homogeneous plate and an individual rod are comparable.

Let us collect the weights in the Korn inequalities for reinforced plates in Table 1. For simplification we set $\rho_{h}=1$, i.e. we consider the value of the coefficients of the weighted norm at a distance from the boundary $\Gamma_{h}^{i}$.

At first sight, the difference in weight is minor, namely only on three positions we observe a reduced weight factor, that is, for $\partial_{2} u_{1}, \partial_{1} u_{2}$ and $u_{3-i}$ in the rods families, $i=1,2$. However, this small change effects crucially the whole system of differential equations in the models for the reinforced plates with welded and isolated periodic families of fibers. As shown in [1], [23, Sect. 2] the homogenization procedure of the plate with welded reinforcing lead to the Dirichlet problem for an elliptic Agmon-Douglis-Nirenberg system with the ADN-indices as in Table 2.

Remark 3.1 Writing a system of partial differential equations $L u=f$ as $\sum_{k} L_{j k} u_{k}=f_{j}$, the ADN-indices denote the order of the differential operator in the component $L_{k j}$. Whenever the system is properly elliptic these indices will determine the coefficients $s_{k}$ and $t_{j}$ such that $L: \prod_{k} W^{m+s_{k}} \rightarrow \prod_{j} W^{m-t_{j}}$ is Fredholm. A consistent table is necessary for ellipticity.

The limiting homogenized system for the isolated reinforcing keeps the ADN-structure in Table 2 but looses the ellipticity! In forthcoming papers (cf. [25]) we shall confirm this

Table 1 Weight factors for $i=1,2$ and $j=1,2,3$

Table 2 The

Agmon-Douglis-Nirenberg indices

\begin{tabular}{lllllll}
\hline & \multicolumn{2}{l}{ welded rods } & & \multicolumn{2}{l}{ disjoint rods } \\
\cline { 6 - 7 } & rods & plate & & rod 1 & rod 2 & plate \\
\hline$\frac{\partial u_{j}}{\partial x_{j}}$ & 1 & $h$ & 1 & 1 & $h$ \\
$\frac{\partial u_{1}}{\partial x_{2}}, \frac{\partial u_{2}}{\partial x_{1}}$ & 1 & $h$ & & $h$ & $h$ & $h$ \\
$\frac{\partial u_{i}}{\partial x_{3}}, \frac{\partial u_{3}}{\partial x_{i}}$ & $h^{2}$ & $h^{2}$ & & $h^{2}$ & $h^{2}$ & $h^{2}$ \\
$u_{1}$ & 1 & 1 & & $h$ & 1 & 1 \\
$u_{2}$ & 1 & 1 & & 1 & $h$ & 1 \\
$u_{3}$ & $h^{2}$ & $h^{2}$ & & $h^{2}$ & $h^{2}$ & $h^{2}$ \\
\hline
\end{tabular}

\begin{tabular}{llll}
\hline$s_{k}$ & $t_{j}$ & & \\
\cline { 2 - 4 } & 1 & 1 & 2 \\
\hline 1 & 2 & 2 & 3 \\
1 & 2 & 2 & 3 \\
2 & 3 & 3 & 4 \\
\hline
\end{tabular}


announcement and also show ways how this situation can be improved using a different physical configuration.

To detect the difference in the norms (2.9) and (2.11) we would like to give a heuristical explanation. To do so, we kill the weight factor $\rho_{h}$ in all norms leaving only factors $h^{q}$. Then we take the particular field

$$
\begin{aligned}
& u_{1}(x)=y_{2}, \quad u_{2}(x)=y_{1}, \quad u_{3}(x)=0 \\
& \varepsilon_{12}(u)=1 \quad \text { and other strain vanish }
\end{aligned}
$$

This field corresponds to shear deformation in longitudinal directions. Then we have

$\begin{array}{lll} & \text { energy } & \text { weighted norm on rods } \\ \text { welded rods } & O(h) & O(h) \\ \text { isolated rods } & O(h) & O\left(h^{2}\right)\end{array}$

This means that the shear deformation of the composite plate in Fig. 1 can be very large, e.g., of order $h^{-1 / 2}$ with the energy of order 1 . In mathematical terms this means that the homogenized system cannot be elliptic and in mechanical terms that the reinforcing with isolated rods does not work in the case of shear longitudinal loading. Any construction engineer can predict the latter and usually the reinforcing elements ought be jointed somehow. Since such jointing needs one extra step in the construction process, it is often avoided.

\subsection{Possible Generalizations}

The obtained results remain valid for more general assumptions on the geometry of the construction $\Xi(h)$. For instance, we fixed the same unit spacing both for the reinforcement of the fibers $\Pi^{1}(h)$ as well as for the fibers $\Pi^{2}(h)$. We can however extend the results to the construction with different spacing $q_{1}$ and $q_{2}$ of the reinforcement. To derive Korn inequalities we introduce the coordinates $\left(\tilde{x}_{1}, \tilde{x}_{2}\right)=\left(x_{1} / a_{1}, x_{2} / a_{2}\right)$ and substitute $\left(u_{1}, u_{2}\right)$ by $\left(u_{1}, \frac{a_{2}}{a_{1}} u_{2}\right)$ in order to keep the form for the replacement $\mathcal{E}(u ; \Xi(h))$ of the elastic energy functional. Indeed, by means of this substitution we keep the form of the term $\left|\frac{\partial u_{1}}{\partial x_{2}}+\frac{\partial u_{2}}{\partial x_{1}}\right|$.

All results also hold for reinforced plates with more general geometrical structures, first in the case when $\pi_{i}$ are Lipschitz domain and, secondly, for a plate with two reclining families of stiff fibres and for a plate with more than two families of fibres (see Fig. 6).

One may also extend the results of the present paper to local periodic thin plates and fibres with more general geometry, such as for slightly curved rods and rods with periodically varying cross-section.
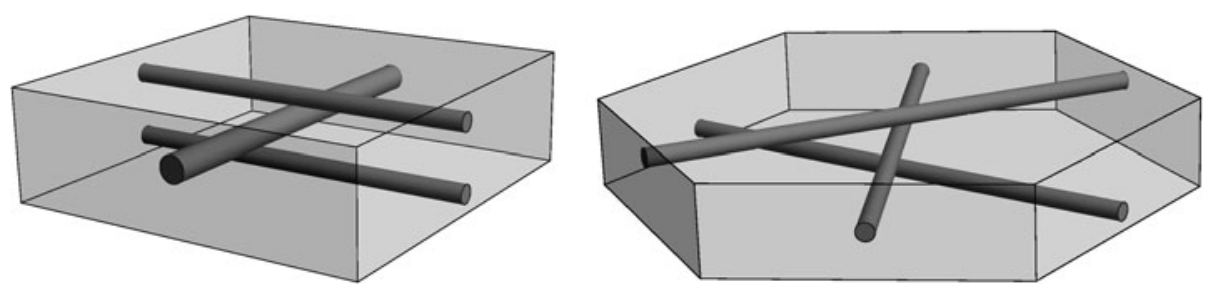

Fig. 6 Other periodicity cells 


\section{Korn Inequalities for a Relatively Rigid Plate}

\subsection{Auxiliary Statements}

To begin with, we recall the result of [13] for Korn inequalities on star-shaped domains.

Lemma 4.1 (See [13]) Let $\Lambda$ be a domain, which is star-shaped with respect to every point in the ball $\mathbb{B}_{R}=\left\{x \in \mathbb{R}^{3} ;|x|<R\right\} \subset \Lambda$. Then for $u \in H^{1}(\Lambda)^{3}$ the following inequalities hold

$$
\begin{aligned}
\left\|\nabla_{x} u ; L_{2}(\Lambda)\right\|^{2} & \leq C_{1} S^{3} R^{-3}\left(\mathcal{E}(u ; \Lambda)+\left\|\nabla_{x} u ; L_{2}\left(\mathbb{B}_{R}\right)\right\|^{2}\right), \\
S^{-2}\left\|u ; L_{2}(\Lambda)\right\|^{2} & \leq C_{2} S R^{-1}\left(\left\|\nabla_{x} u ; L_{2}(\Lambda)\right\|^{2}+R^{-2}\left\|u ; L_{2}\left(\mathbb{B}_{R}\right)\right\|^{2}\right),
\end{aligned}
$$

where $2 S$ is the diameter of $\Lambda$ and $C_{1}, C_{2}$ are constants independent of $S, R$, and $u$.

We will introduce the following norm for a domain $\Lambda$ depending on the parameter $S>0$ :

$$
\|u ; \Lambda\|_{S}^{2}:=\left\|\nabla_{x} u ; L_{2}(\Lambda)\right\|^{2}+S^{-2}\left\|u ; L_{2}(\Lambda)\right\|^{2} .
$$

Combining (4.1) with (4.2), we then come to the estimate

$$
\|u ; \Lambda\|_{S}^{2} \leq C S^{4} R^{-4}\left(\mathcal{E}(u ; \Lambda)+R^{-2}\left\|u ; L_{2}\left(\mathbb{B}_{R}\right)\right\|^{2}\right) .
$$

We derive inequalities of Korn type for the junction $\Xi(h)$ using systematically the resulting estimate (4.4).

Our goal in this section is an inequality for the junction $\Xi$ which corresponds to the estimate (2.4) for the plate $\Omega_{h}$. Korn's inequality (2.4) holds for a vector field supplied with the boundary condition (2.1) on the lateral surface of the plate. However, we consider a vector field defined on the junction $\Xi$ and supplied with the condition (2.5) on the surface $\Gamma(h)$. In order to obtain a Korn type inequality for the junction $\Xi$, we will fix in a more precise way the properties of the domain $\omega$ in the next definition.

Definition 4.1 We call $\omega \subset \mathbb{R}^{2}$ a strong Lipschitz domain if there exists $M \in \mathbb{R}$, such that we can cover the boundary $\partial \omega$ by finitely many open sets $\left\{\mathcal{U}_{i}\right\}_{i=1}^{n}$ with

$$
\omega \cap \mathcal{U}_{i}=\left\{t_{2}^{i}>\varphi_{i}\left(t_{1}^{i}\right)\right\} \cap \mathcal{U}_{i},
$$

where $\left(t_{1}^{i}, t_{2}^{i}\right)$ is a local cartesian coordinate system and where $\varphi_{i}$ satisfies the Lipschitz condition

$$
\left|\varphi_{i}(s)-\varphi_{i}(t)\right| \leq M|s-t|
$$

We start the derivation of a Korn type inequality for the junction $\Xi(h)$ with one auxiliary geometrical statement. Let $\mathcal{G}_{h}^{i, j}, \mathcal{G}^{i}(h)$ denote the projections of $\Pi_{h}^{i, j}, \Pi^{i}(h)$ on the $y$-plane and let $B(P ; r)$ be the circle with radius $r$ and center at the point $P$ in that $y$-plane. Let us introduce the subdomains

$$
\mathcal{H}_{h}^{i, j}(A, B)=\left\{y \in \mathcal{G}_{h}^{i, j} ; y_{i}^{A}<y_{i}<y_{i}^{B}\right\},
$$

where $A=\left(y_{1}^{A}, y_{2}^{A}\right), B=\left(y_{1}^{B}, y_{2}^{B}\right)$. Each domain $\mathcal{H}_{h}^{i, j}(A, B)$ consists of at most finitely many connected components, say $\mathcal{H}_{h}^{i, j, k}(A, B)$. 
Lemma 4.2 There exist $h_{0}>0$ and $C>0$ such that for all $h \in\left(0, h_{0}\right)$ and $P_{0} \in \omega$, $C h<\operatorname{dist}\left(P_{0}, \partial \omega\right)<2 C h$ we can find numbers $i, j, k$ and points $A_{+} \in \omega, A_{-} \notin \omega$, such that $\operatorname{dist}\left(A_{+}, \partial \omega\right)<$ ch and $\operatorname{dist}\left(A_{-}, \partial \omega\right)<$ ch and such that for sufficiently small $\delta>0$ we have

$$
H_{h}^{i, j, k}\left(A_{+}, A_{+}+\delta h\right) \subset B\left(P_{0}, C h\right), \quad H_{h}^{i, j, k}\left(A_{-}, A_{-}-\delta h\right) \cap \omega=\emptyset .
$$

Proof If $P_{0} \in \mathcal{G}_{1} \cup \mathcal{G}_{2}$ the assertion becomes evident. We consider the case where $P_{0} \notin$ $\mathcal{G}_{1} \cup \mathcal{G}_{2}$. Let $Q_{0}=\left(t_{1}^{0}, t_{2}^{0}\right) \in \partial \omega$ be the point such that

$$
\left|Q_{0} P_{0}\right|=\inf _{Q \in \partial \omega}\left|Q P_{0}\right|,
$$

i.e. $\left|Q_{0} P_{0}\right|$ is a distance from $P_{0}$ to $\partial \omega$. Let $\left(t_{1}, t_{2}\right)$ be a coordinate system in the vicinity of $P_{0}$ where the curve $\partial \omega$ is defined by the equality $t_{2}=\varphi\left(t_{1}\right)$ and $\varphi$ satisfies condition (4.5).

There exist a point $Q_{m}=\left(y_{1}^{m}, y_{2}^{m}\right) \in \mathcal{G}_{h}^{m, p} \cap \partial \omega$ in the open set $\mathcal{U} \in\left\{\mathcal{U}_{i}\right\}_{i=1}^{n}$ such that the estimate $\left|t_{1}^{0}-t_{1}^{m}\right|<\sqrt{2} h$ holds, where $\left(t_{1}^{0}, t_{2}^{0}\right)$ and $\left(t_{1}^{m}, t_{2}^{m}\right)$ are the local coordinates of the points $Q_{0}$ and $Q_{m}$. According to the Lipschitz condition in (4.5) with constant $M$, we can find a point $Q_{3-m}=\left(y_{1}^{3-m}, y_{2}^{3-m}\right) \in \mathcal{G}_{h}^{3-m, q} \cap \partial \omega$ such that $Q_{3-m} \in B\left(Q_{m}, h\left(1+M^{2}\right)^{1 / 2}\right)$. Let us select $\mathcal{G}_{h}^{i, j}$. If the segment $P_{0} Q_{0}$, connecting the points $P_{0}$ and $Q_{0}$ and $\mathcal{G}_{h}^{m, p} \cap \omega$ has an acute angle, we put $(i, j)=(m, p)$; otherwise $P_{0} Q_{0}$ and $\mathcal{G}_{h}^{3-m, q} \cap \omega$ form an acute angle and we take $(i, j)=(3-m, q)$. Hence, for sufficiently large $C$ and small $h$ there exists such a point $A \in \omega$ with $\mathcal{G}_{h}^{i, j} \cap B\left(P_{0}, C h\right)=H_{h}^{i, j, k}(A, A+\delta h)$, where $(i, j)=(m, p)$ or $(i, j)=(3-m, q)$ and

$$
\operatorname{dist}\left|A Q_{i}\right| \leq \operatorname{dist}\left|Q_{i} Q_{0}\right|+\operatorname{dist}\left|Q_{0} P_{0}\right|+\operatorname{dist}\left|P_{0} A\right| \leq(M+2 C) h:=c h .
$$

Since the set $\mathcal{U}$ does not depend on $h$, we take $h$ sufficiently small in order to define the point $A_{-}$.

4.2 Korn's Inequality for $\Xi(h)$

Let $H_{0}^{1}(\Xi(h))^{3}$ be the closure of $\left.C_{0}^{\infty} \overline{(\Xi(h)} \backslash\left(\Gamma_{1}(h) \cup \Gamma_{2}(h)\right)\right)^{3}$ with respect to the Sobolev $H(\Xi(h))^{3}$-norm.

Theorem 4.1 For $u \in H_{0}^{1}(\Xi(h))^{3}$ the inequality

$$
|u ; \Xi(h)|_{0}^{2} \leq c \mathcal{E}(u ; \Xi(h)),
$$

is valid where $c$ is a constant independent of $u$ and $h \in\left(0, h_{0}\right]$.

Proof Let $\chi \in C^{\infty}(\mathbb{R})$ be a cut-off function, $\chi(t)=0$ for $t<1$ and $\chi(t)=1$ for $t>2$. Put $\chi_{\mathcal{C} h}(y)=\chi\left((\mathcal{C} h)^{-1} \operatorname{dist}(y, \partial \omega)\right)$ and $\mathcal{O}_{\chi}(\mathcal{C} h):=\operatorname{supp}(\nabla \chi)$. According to $(2.4)$ and the definition of the cut-off function $\chi$ we have

$$
|\chi u ; \Xi(h)|_{0}^{2} \leq c \mathcal{E}(\chi u ; \Xi(h)) \leq c\left(\mathcal{E}(u ; \Xi(h))+h^{-2}\left\|u ; L_{2}\left(\mathbb{O}_{\chi}(\mathcal{C} h)\right)\right\|^{2}\right),
$$

where $\mathbb{O}_{\chi}(\mathrm{Ch}):=\mathcal{O}_{\chi}(\mathrm{C} h) \times(-h / 2, h / 2)$.

In order to estimate the second term in the right-hand side of (4.8) we introduce the domains

$$
L_{h}^{i, j, k}(A, B)=\left\{x=(y, z) \in \Pi_{h}^{i, j}: y \in \mathcal{H}_{h}^{i, j, k}(A, B)\right\},
$$


and the cylinders $\mathbb{T}_{h}(P ; r)=\left\{(y, z):\left(y_{1}, y_{2}\right) \in B(P ; h r), z \in(-h / 2, h / 2)\right\}$, where $B(P ; h r)$ is a ball of radius $r h$ with a center at a point $P$.

Let $P^{(1)}$ be a point in $\mathbb{O}_{\chi}(C h)$ and $P_{0}^{(1)}$ be the projection of $P^{(1)}$ on the plane $z=0$. By Lemma 4.2 we have $\mathcal{G}_{h}^{i, j}$ and points $A_{+}, A_{-}$such that the sets $H_{h}^{i, j, k}\left(A_{+}, A_{+}+\delta h\right)$ and $H_{h}^{i, j, k}\left(A_{-}, A_{-}-\delta h\right)$ satisfy conditions (4.6). Let $L_{h}^{i, j, k}$ correspond to the domain $H_{h}^{i, j, k}$. By Lemma 4.2, for a sufficiently large $C_{0}$, we have $L_{h}^{i, j, k}\left(A_{+}+\delta h\right) \subset \mathbb{T}_{h}\left(P_{0}^{(1)} ; C_{0}\right)$. Let us take a ball $\mathbb{B}_{l h}^{i, j, k} \subset L_{h}^{i, j, k}\left(A_{+}, A_{+}+\delta h\right)$ of radius $l h$ with sufficiently small $l>0$. The cylinder $\mathbb{T}_{h}\left(P_{0}^{(1)} ; C_{0}\right)$ is star-shaped with respect to the ball $\mathbb{B}_{l h}^{i, j, k}$. Hence, by (4.4), we have

$$
\begin{aligned}
\left\|u ; \mathbb{T}_{h}\left(P_{0}^{(1)} ; C_{0}\right)\right\| \|_{h}^{2} & \leq c\left(\mathcal{E}\left(u ; \mathbb{T}_{h}\left(P_{0}^{(1)} ; C_{0}\right)\right)+h^{-2}\left\|u ; L_{2}\left(\mathbb{B}_{l h}^{i, j, k}\right)\right\|^{2}\right) \\
& \leq c\left(\mathcal{E}\left(u ; \mathbb{T}_{h}\left(P_{0}^{(1)} ; C_{0}\right)\right)+h^{-2}\left\|u ; L_{2}\left(L_{h}^{i, j, k}\left(A_{+}, A_{+}+\delta h\right)\right)\right\|^{2}\right) \\
& \leq c\left(\mathcal{E}\left(u ; \mathbb{T}_{h}\left(P_{0}^{(1)} ; C_{0}\right)\right)+\left|u ; L_{h}^{i, j, k}\left(A_{-}, A_{+}+\delta h\right)\right|_{i}^{2}\right) .
\end{aligned}
$$

The last inequality follows from definition (2.6) of the norm $|u ; \cdot|_{i}$. According to (4.9) and Theorem 2.2 we have the estimate

$$
\left\|u ; \mathbb{T}_{h}\left(P_{0}^{(1)} ; C_{0}\right)\right\|_{h}^{2} \leq c\left(\mathcal{E}\left(u ; \mathbb{T}_{h}\left(P_{0}^{(1)} ; C_{0}\right)\right)+\mathcal{E}\left(u ; \Pi_{h}^{i, j}\right)\right) .
$$

We take a next point $P^{(2)} \subset \mathbb{O}_{\chi}(\mathcal{C} h) \backslash \mathbb{T}_{h}\left(P_{0}^{(1)} ; C_{0}\right)$ and find a cylinder where $u$ satisfies the estimate (4.10). Let us continue the process. We define points $P^{(3)}, P^{(4)}, \ldots$ and obtain a system of cylinders which cover the domain $\mathbb{O}_{\chi}(\mathrm{Ch})$. Combining the estimates (4.10) for all those cylinders, we come to

$$
\left\|u ; \mathbb{O}_{\chi}(\mathcal{C} h)\right\|_{h}^{2} \leq c \mathcal{E}(u ; \Xi(h)) .
$$

By (4.8) and (4.11), we have

$$
|\chi u ; \Xi(h)|_{0}^{2} \leq c \mathcal{E}(u ; \Xi(h)) .
$$

To prove (4.7), it remains to find an estimate for the norm $|(1-\chi) u ; \Xi(h)|_{0}^{2}$. Set $\widetilde{\mathbb{O}}_{\chi}(\mathcal{C} h):=(-h / 2, h / 2) \times \operatorname{supp}(1-\chi)$. In this domain we have $\rho_{h}(y)=O\left(h^{1}\right)$. Thus, the following inequality holds:

$$
\begin{aligned}
& |(1-\chi) u ; \Xi(h)|_{0}^{2} \\
& \quad \leq c\left(h^{-2}\left\|u ; L_{2}\left(\mathbb{O}_{\chi}(\mathcal{C} h)\right)\right\|\right. \\
& \left.\left.\quad+\left\|u ; \widetilde{\mathbb{O}}_{\chi}(\mathcal{C} h) \cap\left(\Pi^{1}(h) \cup \Pi^{2}(h)\right)\right\|_{h}+\| u ; \widetilde{\mathbb{O}}_{\chi}(\mathcal{C} h) \cap \Omega_{h}\right) \|_{h}\right) .
\end{aligned}
$$

The required estimate for the first two terms on the right-hand side of (4.13) follows from (4.11), (2.4). In order to estimate the third term we cover the domain $\mathcal{O}_{\chi}(\mathrm{Ch})$ by a system of circles $\mathcal{T}_{h}^{(j)}, j=1, \ldots, J$, with radii $R h$ where $R$ is a sufficiently large number. We project those circles onto the curve $\partial \omega$. Let $\mathcal{S}_{h}^{(j)}$ be a union of a circle $\mathcal{T}_{h}^{(j)}$ and all points situated between $\mathcal{T}_{h}^{(j)}$ and the projection of $\mathcal{T}_{h}^{(j)}$ on $\partial \omega$.

We prove by contradiction that $\mathcal{S}_{h}^{(j)}$ is star-shaped with respect to the circle $\mathcal{T}_{h}^{(j)}$. Suppose that $\tau^{-} \in \mathcal{S}_{h}^{(j)} \backslash \mathcal{T}_{h}^{(j)}, \tau^{+} \in \mathcal{T}_{h}^{(j)}$ and $\left(\tau^{-}, \tau^{+}\right) \nsubseteq \mathcal{T}_{h}^{(j)}$. Hence, we have a point $\tau^{0}$ such that 
$\tau^{0} \in\left(\tau^{-}, \tau^{+}\right)$and $\tau^{0} \in \partial \omega$. For sufficiently small $h$, there exists a coordinate system $\left(t_{1}, t_{2}\right)$ where the curves $\partial \omega \cap \mathcal{S}_{h}^{(j)}$ are defined by the equality $t_{2}=\varphi\left(t_{1}\right)$ and $\varphi$ satisfies (4.5). The Lipschitz condition (4.5) implies

$$
\left|\tau_{2}^{-}-\tau_{2}^{0}\right| \leq M\left|\tau_{1}^{-}-\tau_{1}^{0}\right|
$$

where $\tau_{i}^{-}, \tau_{i}^{0}$ are coordinates of the points $\tau^{-}, \tau^{0}$ in the coordinate system $\left(t_{1}, t_{2}\right)$. According to the definition of the domain $\mathcal{S}_{h}^{(j)}$ and the points $\tau^{-}, \tau^{0}$ we have the geometrical relation

$$
\frac{R}{\mathcal{C}+R} \geq \frac{\left|\tau_{1}^{+}-\tau_{1}^{-}\right|}{\left|\tau_{2}^{+}-\tau_{2}^{-}\right|} \geq \frac{\left|\tau_{1}^{0}-\tau_{1}^{-}\right|}{\left|\tau_{2}^{0}-\tau_{2}^{-}\right|} .
$$

By (4.14) and (4.15), $(R+\mathcal{C}) R^{-1} \geq M^{-1}$ for sufficiently large $\mathcal{C}$ and, therefore, we have come to a contradiction. Thus, the domain $\mathcal{S}_{h}^{(j)}$ is star-shaped with respect to the circle $\mathcal{T}_{h}^{(j)}$ and consequently the domain $\mathbb{S}_{h}^{(j)}=\mathcal{S}_{h}^{(j)} \times(-h / 2, h / 2)$ is star-shaped with respect to the cylinder $\mathbb{T}_{h}^{(j)}=\mathcal{T}_{h}^{(j)} \times(-h / 2, h / 2)$. Let $\mathbb{B}_{h}^{(j)}$ be a ball of radius $r h$, where $r$ is a number such that $\mathbb{B}_{h}^{(j)} \subset \mathbb{T}_{h}^{(j)}$. By (4.4), we have

$$
\left\|u ; \mathbb{S}_{h}^{(j)}\right\|_{h}^{2} \leq C\left(\mathcal{E}\left(u ; \mathbb{S}_{h}^{(j)}\right)+h^{-2}\left\|u ; L_{2}\left(\mathbb{B}_{h}^{(j)}\right)\right\|^{2}\right) .
$$

We sum the inequalities (4.16) over all $j=1, \ldots, J$ and obtain

$$
\left.\| u ; \widetilde{\mathbb{O}}_{\chi}(\mathcal{C} h) \cap \Omega_{h}\right) \|_{h} \leq C\left(\mathcal{E}\left(u ; \mathbb{O}_{\chi}(\mathcal{C} h) \cap \Omega_{h}\right)+\left\|u ; \mathbb{O}_{\chi}(\mathcal{C} h) \cap \Omega_{h}\right\|_{h}\right) .
$$

According to (4.13), (4.11) and (4.17) we find that

$$
|(1-\chi) u ; \Xi(h)|_{0}^{2} \leq c \mathcal{E}(u ; \Xi(h)) .
$$

Combining (4.12) and (4.18) completes the proof.

From the obvious inequality

$$
\min \{1, \gamma\} \mathcal{E}(u ; \Xi(h)) \leq \mathcal{E}_{\gamma}(u ; \Xi(h)),
$$

we obtain the following result.

Corollary 4.1 For $u \in H_{0}^{1}(\Xi(h))^{3}$, the following estimate is valid

$$
\min \{1, \gamma\}|u ; \Xi(h)|_{0}^{2} \leq c \mathcal{E}_{\gamma}(u ; \Xi(h)),
$$

where the constant $c$ is independent of the vector field $u$ and the parameters $\gamma>0, h \in$ $\left(0, h_{0}\right]$.

\subsection{The Estimate in (4.19) is Optimal}

In order to verify that (4.19) is asymptotically sharp for $\gamma>1$ we define the vector field

$$
U(x)=\left(e_{1}+e_{2}+e_{3}\right) X_{h}(y)-z \frac{\partial X_{h}}{\partial y_{1}}(y) e_{1}-z \frac{\partial X_{h}}{\partial y_{2}}(y) e_{2}
$$


where $X_{h} \in C^{\infty}\left(\mathbb{R}^{2}\right)$ is a cut-off function, $X_{h}(y)=1$ for $y \in \Omega_{h}$ and $X_{h}(y)=0$ for $y \in \Gamma(h)$.

The field in (4.20) satisfies the equalities $\varepsilon_{3 j}(U)=\varepsilon_{j 3}(U)=0, j=1,2,3$ and

$$
\begin{aligned}
& \varepsilon_{11}(U)=\frac{\partial X_{h}}{\partial y_{1}}(y)-z \frac{\partial^{2} X_{h}}{\partial y_{1}^{2}}(y), \quad \varepsilon_{22}(U)=\frac{\partial X_{h}}{\partial y_{2}}(y)-z \frac{\partial^{2} X_{h}}{\partial y_{2}^{2}}(y), \\
& \varepsilon_{12}(U)=\varepsilon_{21}(U)=\frac{1}{2} \frac{\partial X_{h}}{\partial y_{1}}(y)+\frac{1}{2} \frac{\partial X_{h}}{\partial y_{2}}(y)+z \frac{\partial^{2} X_{h}}{\partial y_{1} \partial y_{2}}(y) .
\end{aligned}
$$

Hence,

$$
\mathcal{E}_{\gamma}(U ; \Xi(h))=O\left(\gamma^{0} h^{2} h^{-2}\right)=O\left(\gamma^{0} h^{0}\right) .
$$

The factor $h^{2}$ appears in (4.21) due to the size of the 3d-Lebesgue measure

$$
\operatorname{mes}_{3}\left(\operatorname{supp}\left|\nabla X_{h}\right|\right)=O\left(h^{2}\right) \text {. }
$$

The factor $h^{-2}$ arises from the next relation for $k=1$ :

$$
\left|\nabla^{k} X_{h}(y)\right|=O\left(h^{-k}\right)
$$

All the terms in the expressions $|U ; \Xi(h)|_{0}$ (see (2.3)) have the same order $h^{0}$ as $\mathcal{E}_{\gamma}(U ; \Xi(h))$. The relations

$$
\begin{aligned}
& \int_{\Xi(h)} \frac{h^{2}}{\rho_{h}^{4}}\left|U_{3}\right|^{2} d y d z=h^{2} O\left(h^{1} h^{-3}\right)=O\left(h^{0}\right), \\
& \int_{\Xi(h)} \frac{1}{\rho_{h}^{2}}\left|U_{i}\right|^{2} d y d z=O\left(h^{1} h^{-1}\right)=O\left(h^{0}\right)
\end{aligned}
$$

follow from

$$
\int_{\omega} \rho_{h}^{-k}(y) d y=O\left(h^{-k+1}\right)
$$

with $k=2$ and $k=4$. The orders, analogous to (4.24), for the other terms of $|U ; \Xi(h)|_{0}$ follow from (4.22) with $k=1,2$, (4.25) with $k=2$, and the relation $|z|=O\left(h^{1}\right)$ for $z \in \Xi(h)$.

\section{An Improved Weighted Korn Inequality for the Matrix}

\subsection{Korn's Inequality for $\Theta(h)$}

In the previous section we have obtained the Korn inequality (4.19) and have proved that it is asymptotically sharp for $\gamma>1$ on the junction $\Xi(h)$ and, in particular, on the matrix $\Theta(h)$. In this section we derive the Korn inequality for the matrix $\Theta(h)$ which is asymptotically sharp in the case $1>\gamma>0$.

Let $\Lambda(h)$ be an open subset of $\Xi(h)$ and let us introduce in the space $H^{1}(\Lambda(h))^{3}$ the weighted anisotropic norm 


$$
\begin{aligned}
|u ; \Lambda(h)|_{\gamma, \bullet}:= & \left(\int _ { \Lambda ( h ) } \left\{\min \{1, \gamma\}\left(\left|\frac{\partial u_{1}}{\partial y_{1}}\right|^{2}+\left|\frac{\partial u_{2}}{\partial y_{2}}\right|^{2}+\left|\frac{\partial u_{3}}{\partial z}\right|^{2}\right)\right.\right. \\
& +h^{2} \beta_{1}(h, \gamma, y)\left(\left|\frac{\partial u_{1}}{\partial y_{2}}\right|^{2}+\left|\frac{\partial u_{2}}{\partial y_{1}}\right|^{2}+\left|\frac{\partial u_{1}}{\partial z}\right|^{2}+\left|\frac{\partial u_{3}}{\partial y_{1}}\right|^{2}+\left|\frac{\partial u_{2}}{\partial z}\right|^{2}+\left|\frac{\partial u_{3}}{\partial y_{2}}\right|^{2}\right) \\
& \left.\left.+\beta_{1}(h, \gamma, y)\left(\left|u_{1}\right|^{2}+\left|u_{2}\right|^{2}\right)+h^{2} \beta_{2}(h, \gamma, y)\left|u_{3}\right|^{2}\right\} d y d z\right)^{1 / 2}
\end{aligned}
$$

where $\beta_{1}$ and $\beta_{2}$ are weight factors which depend on the geometrical parameter $h$, the physical parameter $\gamma$ and the variable $y$.

Lemma 5.1 For $u \in H_{0}^{1}(\Xi(h))^{3}$ the following inequality holds:

$$
|u ; \Theta(h)|_{\gamma, \bullet}^{2} \leq c \mathcal{E}_{\gamma}(u ; \Xi(h))
$$

where $\beta_{1}, \beta_{2}$ defined in (3.2), $c$ is a constant independent of $u, \gamma$ and $h \in\left(0, h_{0}\right]$.

Proof Away from the lateral boundary the medium is periodic. The standard periodicity cell implies

$$
S(h)=\left\{(y, z) ;\left|y_{i}\right|<\frac{1}{2} h \text { and }|z|<\frac{1}{2} h\right\}
$$

We consider the cells

$$
S^{(m)}(h):=\{(y, z) ;(y-m h, z) \in S(h)\}
$$

for all $m \in \mathbb{Z}^{2}$ with $S^{(m)}(h) \subset \Omega_{h}$.

Let us choose circular cylinders $G_{i}(h):=\Pi^{i, q(m)}(h) \cap S^{(m)}(h), i=1,2$, directed parallel to the to the $y_{3-i}$-axes, where $q(m)=\left\{j: \Pi^{i, j}(h) \cap S^{(m)} \neq \emptyset\right\}$ (see Fig. 3). We set the coordinate origin in the mass center of the cylinder $G_{1}(h)$. Let $Q(h)$ be the set

$$
Q(h)=S^{(m)}(h) \backslash\left(G_{1}(h) \cup G_{2}(h)\right) .
$$

On the cell $S^{(m)}(h)$ the vector $u$ admits the representation

$$
u(x)=u^{\perp}(x)+d(x) a,
$$

where $a \in \mathbb{R}^{6}$ and the vector $u^{\perp}$ satisfies the orthogonality condition

$$
\int_{Q(h)} d(x)^{\top} u^{\perp}(x) d x=0 .
$$

Let $s(h)$ denote the distance from the center of the parallelepiped $S(h)$ to the lateral surface $\Gamma(h)$ of $\Xi(h)$. In order to estimate the norm of the second term in (5.3) we introduce the number $\kappa_{h}=s(h)-h$. The inequality

$$
c \rho_{h}(y) \leq \kappa_{h} \leq C \rho_{h}(y)
$$

holds because of the definitions of the function $\rho_{h}$ and the number $\kappa_{h}$. 
By (5.1) and (1.4), we have

$$
\begin{aligned}
& |d(\cdot) a ; Q(h)|_{\gamma, \bullet}^{2} \\
& \quad \leq c h^{3}\left(\mu_{1}\left(\left(\left|a_{1}\right|^{2}+\left|a_{2}\right|^{2}\right)+h^{2}\left(\left|a_{4}\right|^{2}+\left|a_{5}\right|^{2}+\left|a_{6}\right|^{2}\right)\right)\right. \\
& \left.\quad+h^{2} \mu_{2}\left(\left|a_{3}\right|^{2}+h^{2}\left(\left|a_{4}\right|^{2}+\left|a_{5}\right|^{2}\right)\right)\right) \\
& \quad \leq c^{3}\left(\mu_{1}\left(\left(\left|a_{1}\right|^{2}+\left|a_{2}\right|^{2}\right)+h^{2}\left(\left|a_{4}\right|^{2}+\left|a_{5}\right|^{2}+\left|a_{6}\right|^{2}\right)\right)+h^{2} \mu_{2}\left|a_{3}\right|^{2}\right)
\end{aligned}
$$

where

$$
\mu_{1}(h, \gamma)=\min \left\{\left(\kappa_{h}\right)^{-2}, \gamma h^{-2}\right\}, \quad \mu_{2}(h, \gamma)=\min \left\{\left(\kappa_{h}\right)^{-4}, \gamma h^{-4}\right\} .
$$

Taking the scalar product of (5.3) with the first unit vector $e_{1}$ and integrating it over $\Sigma_{1}(h)=\partial G_{1}(h) \backslash \partial S(h)$, we obtain

$$
\left|\Sigma_{1}(h)\right| a_{1}=\int_{\Sigma_{1}(h)}\left(u_{1}(x)-u_{1}^{\perp}(x)\right) d s_{x} .
$$

We have used the symmetry of the circular cylinder $G_{1}(h)$ while calculating the left-hand side in (5.7).

Since $\left|\Sigma_{1}(h)\right|=O\left(h^{2}\right)$, we arrive by the Cauchy-Bunyakowsky-Schwarz inequality at the estimate

$$
\left|a_{1}\right|^{2} \leq c h^{-2}\left(\kappa_{h}^{2} \int_{\Sigma_{1}(h)} \rho_{h}^{-2} u_{1}(x)^{2} d s_{x}+\int_{\Sigma_{1}(h)} u_{1}^{\perp}(x)^{2} d s_{x}\right)
$$

To process the right-hand side, we use the well-known trace inequality

$$
h \int_{\partial Q(h)}|U|^{2} d s_{x} \leq c \int_{Q(h)}\left\{h^{2}|\nabla U|^{2}+|U|^{2}\right\} d x
$$

for a vector-function $U \in H^{1}(Q(h))$ (see, e.g. [21], Proposition 1.2.8). From (5.9) with $U=u_{1}^{\perp}$ it follows that

$$
h \int_{\Sigma_{1}(h)}\left|u_{1}^{\perp}\right|^{2} d s_{x} \leq c \int_{Q(h)}\left\{h^{2}\left|\nabla u_{1}^{\perp}\right|^{2}+\left|u_{1}^{\perp}\right|^{2}\right\} d x=c h^{2} \mid\left\|u^{\perp} ; Q(h)\right\|_{h}^{2} .
$$

Equality (5.4) implies the estimate

$$
\left\|u^{\perp} ; Q(h)\right\|_{h}^{2} \leq c \mathcal{E}\left(u^{\perp} ; Q(h)\right)
$$

(see, for instance [21, Theorem 2.3.3], [23, Proposition 2.1]). Hence, see [21, Proposition 3.4.12],

$$
\int_{\Sigma_{1}(h)}\left|u_{1}^{\perp}\right|^{2} d s_{x} \leq \operatorname{ch} \mathcal{E}\left(u^{\perp} ; Q(h)\right) .
$$

By the trace inequality (5.9) for $U=\rho_{h}^{-1} u_{1}$, we obtain

$$
\kappa_{h}^{2} \int_{\Sigma_{1}(h)} \rho_{h}^{-2} u_{1}(x)^{2} d s_{x} \leq c h^{-1} \kappa_{h}^{2}\left|u ; G_{1}(h)\right|_{1}^{2} .
$$


Thus, in accordance with (5.8), (5.12), (5.13) and the identity

$$
\mathcal{E}_{\gamma}\left(u^{\perp} ; Q(h)\right)=\mathcal{E}_{\gamma}(u ; Q(h))
$$

we find

$$
\left|a_{1}\right|^{2} \leq c h^{-3}\left(\kappa_{h}^{2}\left|u ; G_{1}(h)\right|_{1}^{2}+h^{2} \gamma^{-1} \mathcal{E}_{\gamma}\left(u^{\perp} ; Q(h)\right)\right),
$$

where

$$
\mathcal{E}_{\gamma}(u ; Q(h)):=\gamma \mathcal{E}(u ; Q(h)) .
$$

In order to estimate $a_{3}$ we take the scalar product of (5.3) with $e_{3}$ and arrive at the inequality

$$
\left|a_{3}\right|^{2} \leq \operatorname{ch}^{-2}\left(\kappa_{h}^{4} \int_{\Sigma_{1}(h)} \rho_{h}^{-4} u_{3}(x)^{2} d s_{x}+\int_{\Sigma_{1}(h)} u_{3}^{\perp}(x)^{2} d s_{x}\right)
$$

(cf. (5.8)). By analogy to (5.14), from the definition of the norm $|\cdot|_{1}$ in (2.6) and (5.9) for $U=u_{3}^{\top}$ and $U=\rho_{h}^{-2} u_{3}$, one obtains the formula

$$
\left|a_{3}\right|^{2} \leq \operatorname{ch}^{-5}\left(\kappa_{h}^{4}\left|u ; G_{1}(h)\right|_{1}^{2}+h^{4} \gamma^{-1} \mathcal{E}_{\gamma}(u ; Q(h))\right) .
$$

We find an estimate for $a_{6}$ by taking the scalar product of (5.3) with $x_{2} e_{1}$. In the same way as in (5.8) we have

$$
\left|a_{6}\right|^{2} \leq c\left(\int_{\Sigma_{1}(h)} x_{2}^{2} d s_{x}\right)^{-1}\left(\kappa_{h}^{2} \int_{\Sigma_{1}(h)} \rho_{h}^{-2} u_{1}(x)^{2} d x+\int_{\Sigma_{1}(h)} u_{1}^{\perp}(x)^{2} d s_{x}\right) .
$$

Hence,

$$
\left|a_{6}\right|^{2} \leq c h^{-5}\left\{\kappa_{h}^{2}\left|u ; G_{1}(h)\right|_{1}^{2}+h^{2} \gamma^{-1} \mathcal{E}_{\gamma}(u ; Q(h))\right\} .
$$

We have used the relation $c h^{2} \leq x_{2}^{2} \leq C h^{2}$ for $x \in \Sigma_{1}(h)$.

Taking the scalar product of (5.3) with $x_{2} e_{1}$, we obtain the inequality

$$
\left|a_{5}\right|^{2} \leq c h^{-5}\left\{\kappa_{h}^{2}\left|u ; G_{1}(h)\right|_{1}^{2}+h^{2} \gamma^{-1} \mathcal{E}_{\gamma}(u ; Q(h))\right\},
$$

similarly to (5.17).

In order to estimate the components $a_{2}$ and $a_{4}$ we use the trace inequality for the cylinder $G_{2}$. Let the center of the circular cylinder $G_{2}$ have the coordinates $\delta_{1}(h), \delta_{2}(h), \delta_{3}(h)$ and let us introduce the coordinates $\left(x_{1}^{\prime}, x_{2}^{\prime}, x_{3}^{\prime}\right)=\left(x_{1}+\delta_{1}(h), x_{2}+\delta_{2}(h), x_{3}+\delta_{3}(h)\right)$. For the first term in the right-hand side of the representation

$$
u\left(x^{\prime}\right)=u^{\perp}\left(x^{\prime}\right)+d\left(x^{\prime}\right) a .
$$

we have the estimate

$$
\int_{\Sigma_{2}(h)}\left|u_{2}^{\perp}\right|^{2} d s_{x} \leq \operatorname{ch\mathcal {E}}\left(u^{\perp} ; Q(h)\right)
$$

(see the derivation of (5.12)).

Let us turn to the second term in (5.19). The vector $d(x) a$ in the new coordinates $x^{\prime}$ has the form

$$
d\left(x^{\prime}\right)\left(a_{1}^{\prime}, a_{2}^{\prime}, a_{3}^{\prime}, a_{4}, a_{5}, a_{6}\right)^{\top}
$$


where

$$
\begin{aligned}
& a_{1}^{\prime}=a_{1}+2^{-1 / 2} \delta_{3} a_{5}-2^{-1 / 2} \delta_{2} a_{6}, \\
& a_{2}^{\prime}=a_{2}-2^{-1 / 2} \delta_{3} a_{4}+2^{-1 / 2} \delta_{1} a_{6}, \\
& a_{3}^{\prime}=a_{3}+2^{-1 / 2} \delta_{2} a_{4}-2^{-1 / 2} \delta_{1} a_{5} .
\end{aligned}
$$

Take the scalar product (5.3) with $e_{2}$ and with $e_{2} x_{3}^{\prime}$, and then integrate the results over $\Sigma_{2}(h)=\partial \mathcal{G}_{2}(h) \backslash \partial S(h)$. We obtain the equalities

$$
\begin{aligned}
\left|\Sigma_{2}(h)\right|\left(a_{2}-2^{-1 / 2} \delta_{3} a_{4}+2^{-1 / 2} \delta_{1} a_{6}\right) & =\int_{\Sigma_{2}(h)}\left(u_{2}\left(x^{\prime}\right)-u_{2}^{\perp}\left(x^{\prime}\right)\right) d s_{x^{\prime}}, \\
a_{4} \int_{\Sigma_{2}(h)} x_{3}^{\prime 2} d s_{x^{\prime}} & =-2^{1 / 2} \int_{\Sigma_{2}(h)} x_{3}^{\prime}\left(u_{2}\left(x^{\prime}\right)-u_{2}^{\perp}\left(x^{\prime}\right)\right) d s_{x^{\prime}} .
\end{aligned}
$$

First we consider (5.21). Analogously to (5.16) one verifies that

$$
\left|a_{4}\right|^{2} \leq c\left(\int_{\Sigma_{2}(h)} x_{3}^{\prime 2} d s_{x^{\prime}}\right)^{-1} \kappa_{h}^{2} \int_{\Sigma_{2}(h)}\left(\rho_{h}^{-2} u_{2}\left(x^{\prime}\right)^{2}+u_{2}^{\perp}\left(x^{\prime}\right)^{2}\right) d s_{x^{\prime}} .
$$

Since $c h^{2} \leq x_{3}^{\prime 2} \leq C h^{2}$ for $x \in \Sigma_{2}(h)$, just as in (5.17) we have

$$
\left|a_{4}\right|^{2} \leq c h^{-5}\left(\kappa_{h}^{2}\left|u ; G_{2}(h)\right|_{2}^{2}+h^{2} \gamma^{-1} \mathcal{E}_{\gamma}(u ; Q(h))\right) .
$$

In order to estimate $a_{2}$, we use (5.17) and (5.22). Since $\left|\Sigma_{1}(h)\right|=O\left(h^{2}\right)$, it follows from (5.20) that

$$
\left|a_{2}\right|^{2} \leq c\left(h^{-2}\left(\kappa_{h}^{2} \int_{\Sigma_{2}(h)}\left(\rho_{h}^{-2} u_{2}(x)^{2}+u_{2}^{\perp}(x)^{2}\right) d s_{x}\right)^{2}+\left|a_{4}\right|^{2} \delta_{3}^{2}+\left|a_{6}\right|^{2} \delta_{1}^{2}\right) .
$$

The first term has the same form as the right-hand side of (5.8) and therefore it allows the estimate

$$
\begin{aligned}
& h^{-2}\left(\kappa_{h}^{2} \int_{\Sigma_{2}(h)} \rho_{h}^{-2} u_{2}(x)^{2} d s_{x}+\int_{\Sigma_{2}(h)} u_{2}^{\perp}(x)^{2} d s_{x}\right)^{2} \\
& \leq c h^{-3}\left(\kappa_{h}^{2}\left|u ; G_{1}(h)\right|_{2}^{2}+h^{2} \gamma^{-1} \mathcal{E}_{\gamma}(u ; Q(h))\right) .
\end{aligned}
$$

By (5.22), (5.17) and the relations $\left|\delta_{j}(h)\right| \leq c h$ for $j=1,2,3$ we have

$$
\left|a_{4}\right|^{2} \delta_{3}^{2}+\left|a_{6}\right|^{2} \delta_{1}^{2} \leq c h^{-3}\left(\kappa_{h}^{2}\left|u ; G_{1}(h)\right|_{1}^{2}+\kappa_{h}^{2}\left|u ; G_{2}(h)\right|_{2}^{2}+h^{2} \gamma^{-1} \mathcal{E}_{\gamma}(u ; Q(h))\right) .
$$

The inequalities (5.23), (5.24), (5.25) imply

$$
\left|a_{2}\right|^{2} \leq c h^{-3}\left(\kappa_{h}^{2}\left|u ; G_{1}(h)\right|_{1}^{2}+\kappa_{h}^{2}\left|u ; G_{2}(h)\right|_{2}^{2}+h^{2} \gamma^{-1} \mathcal{E}_{\gamma}(u ; Q(h))\right) .
$$

We replace the terms $\left|a_{k}\right|$ in (5.5) by their bounds in (5.14), (5.15), (5.17), (5.18), (5.22), (5.26) and obtain the inequality 


$$
\begin{aligned}
|d a ; Q(h)|_{\gamma, \bullet}^{2} \leq c\{ & \mu_{1}(h, \gamma)\left(R_{h}^{2}\left(\left|u ; G_{1}(h)\right|_{1}^{2}+\left|u ; G_{2}(h)\right|_{2}^{2}\right)+h^{2} \gamma^{-1} \mathcal{E}_{\gamma}(u ; Q(h))\right) \\
& \left.+\mu_{2}(h, \gamma)\left(R_{h}^{4}\left|u ; G_{1}(h)\right|_{1}^{2}+h^{4} \gamma^{-1} \mathcal{E}_{\gamma}(u ; Q(h))\right)\right\}
\end{aligned}
$$

Thus, we have processed the second term in the representation (5.3). According to the definitions in (4.3), (5.1) of the norms $\||\cdot ; Q(h)|\|_{h}^{2},|\cdot ; Q(h)|_{\gamma, \bullet}^{2}$ and the formula in (5.10) it holds that

$$
\left|u^{\perp} ; Q(h)\right|_{\gamma, \bullet}^{2} \leq c\left(\min \{1, \gamma\} \mathcal{E}(u ; Q(h))+\gamma\|\| u^{\perp} ; Q(h) \|_{h}\right) \leq c \mathcal{E}_{\gamma}(u ; Q(h)) .
$$

We sum (5.27), (5.28) over all $Q(h) \subset \Xi(h)$ and we arrive by (5.6) at the inequality

$$
|u ; \Theta(h)|_{\gamma, \bullet}^{2} \leq c\left(\sum_{q}\left(\left|u ; \Pi^{1, q}(h)\right|_{1}^{2}+\left|u ; \Pi^{2, q}(h)\right|_{2}^{2}\right)+\mathcal{E}_{\gamma}(u ; \Omega(h))\right) .
$$

Now the estimate in (5.2) follows from (2.7) and (5.29).

According to the evident relation $h^{2} \rho_{h}^{-2} \leq 1$ we have

$$
\begin{aligned}
h^{2} \min \left\{\rho_{h}^{-2}, \gamma h^{-2}\right\} & \leq \min \{1, \gamma\}, \\
\min \left\{\rho_{h}^{-2}, \gamma h^{-2}\right\} & \geq \rho_{h}^{-2} \min \{1, \gamma\}, \\
\min \left\{\rho_{h}^{-4}, \gamma h^{-2}\right\} & \geq \rho_{h}^{-4} \min \{1, \gamma\} .
\end{aligned}
$$

Hence, one can introduce the norm (3.1) and combine Corollary 4.1 and Lemma 5.1 into one Theorem 3.1. Note that the norms $|\cdot ; \Theta(h)|_{\gamma, \bullet}$ and $|\cdot ; \Theta(h)|_{\gamma, 0}$ differ only by factors on the terms $\left|\frac{\partial u_{1}}{\partial y_{2}}\right|^{2}$ and $\left|\frac{\partial u_{2}}{\partial y_{1}}\right|^{2}$.

\subsection{The Weight Distribution for the Composite is Optimal: Example 1}

Let us construct particular vector fields in order to verify that the estimate (3.3) is asymptotically sharp. In the case of $\gamma>1$ we have the equality $\min \{1, \gamma\}|u ; \Theta(h)|_{\gamma, 0}=|u ; \Theta(h)|_{0}$ and a suitable vector field has the form (4.20).

If $\rho_{h}^{-4} \geq \gamma h^{-4}$, we use the field

$$
U(x)=\left(1-\chi\left(h^{-1} r_{0}\left|x-x_{0}\right|\right)\right)\left(e_{1}+e_{2}+e_{3}\right),
$$

where $\chi$ is a cut-off function defined in the proof of Theorem 4.1 We take $r_{0}$ and $x_{0}$ so that $\operatorname{supp}\left(x \mapsto 1-\chi\left(h^{-1} r_{0}\left|x-x_{0}\right|\right)\right)=\mathbb{B}_{h}$ belongs to one periodicity cell $S(h), \mathbb{B}_{h} \subset Q(h)$ and $\operatorname{dist}\left(\mathbb{B}_{h}, \partial \Omega_{h}\right) \leq C h$. On the one hand, we have $\mathcal{E}_{\gamma}(U ; \Xi(h))=O\left(\gamma h^{-2} h^{3}\right)=O\left(\gamma h^{1}\right)$. On the other hand, all terms in the right-hand side of (3.3) also are of order $\gamma h^{1}$. Note, that in (3.1) the weight factor $\rho_{h}(y)$ has an influence only on the factors $\beta_{1}(h, \gamma, y)$ and $\beta_{2}(h, \gamma, y)$. Therefore, the field (5.30) verifies the terms of (3.1) with the factor $\min \{1, \gamma\}$ for all $x \in \Xi(h)$.

\subsection{The Weight Distribution for the Composite is Optimal: Example 2}

In the case of $\rho_{h}^{-2} \leq \gamma h^{-2}$ we introduce the field

$$
U(y)=h^{-1} w_{3}(y) e_{3}+w_{1}(y) e_{1}+w_{2}(y) e_{2}-z h^{-1}\left(\frac{\partial w_{3}(y)}{\partial y_{1}} e_{1}+\frac{\partial w_{3}(y)}{\partial y_{2}} e_{2}\right),
$$


where the smooth functions $w_{1}, w_{2}, w_{3}$ satisfy for $0<\alpha<1$ the following:

$$
w_{j}(y)=0 \quad \text { and } \quad \nabla w_{3}=0 \quad \text { for } y \in\left\{y \in \mathbb{R}^{2} ; \operatorname{dist}(y, \omega)<h^{\alpha}\right\} .
$$

We have $\mathcal{E}_{\gamma}(U ; \Xi(h))=O\left(h^{1}\right)$. All integrals in the definition in (3.1) of the norm $|U ; \Xi(h)|_{\gamma, 0}^{2}$ have the same order $h^{1}$.

\subsection{The Weight Distribution for the Composite is Optimal: Example 3}

Let us consider the case when both $\gamma h^{-4}<\rho_{h}^{-4}$ and $\gamma h^{-2}<\rho_{h}^{-2}$ hold. In order to verify that the estimate (3.3) is asymptotically sharp, we construct different fields for each term of the norm (3.1). We introduce the field

$$
U(y)=\left(1-\chi\left(r_{0} h^{-1} \gamma^{-1 / 2}\left|y-y_{0}\right|\right)\right) e_{3}
$$

for the $u_{3}$-terms in (3.1). The support $\mathbb{C}_{h, \gamma}$ of the cut-off function $1-\chi\left(r_{0} h^{-1} \gamma^{-1 / 2}\left|y-y_{0}\right|\right)$ is a cylinder having a base with diameter $4 r_{0}^{-1} h^{1} \gamma^{1 / 2}$ and height $h$. It follows from the inequality $\gamma h^{-2}<\rho_{h}^{-2}$ that $\gamma^{1 / 2}<h \rho_{h}<1$. Hence we can take $r_{0}$ and $y_{0}$ in (5.32) such that $\mathbb{C}_{h, \gamma}$ belongs to one periodicity cell $S(h)$ and $\mathbb{C}_{h, \gamma} \subset Q(h)$. We obtain $E_{\gamma}(U, \Xi(h))=$ $O\left(\gamma^{1} h^{3} \gamma^{1} h^{-1} \gamma^{-1}\right)=O(h \gamma)$. The terms with $\left|\frac{\partial U_{3}}{\partial y_{j}}\right|$ in the norm $|U ; \Xi(h)|_{\gamma, 0}$ have the same order $h \gamma$. To verify that the estimate (3.3) is asymptotically sharp for terms with $\left|U_{3}\right|$, we take $r_{0}$ and $y_{0} \operatorname{such}$ that $\operatorname{supp}\left(x \mapsto \chi\left(r_{0} h^{-1}\left|y-y_{0}\right|\right)\right)$ belongs to a cell $S(h)$ with $\operatorname{dist}\left(S(h), \partial \Omega_{h}\right) \leq C h$. Then we use the same relation $O\left(h^{3} \gamma^{1} h^{-4} h^{2}\right)=O(h \gamma)$ for those terms in the norm $|U ; \Xi(h)|_{\gamma, 0}$. Let us consider the field

$$
U(x)=\left(1-\chi\left(r_{0} h^{-1}\left|y-y_{0}\right|\right)\right)\left(1-\chi\left(r_{1} h^{-1} \gamma^{-1 / 2}\left|z-z_{0}\right|\right)\right)\left(e_{1}+e_{2}\right) .
$$

This field is equal to zero everywhere in $\Xi(h)$ except in a cylinder with the base diameter $4 r_{0}^{-1} h^{1}$ and height $4 h^{1} \gamma^{1 / 2}$. We have

$$
E_{\gamma}(U, \Xi(h))=O\left(\gamma^{1} h^{3} \gamma^{1 / 2} \gamma^{-1} h^{-2}\right)=O\left(h \gamma^{1 / 2}\right) .
$$

The terms of (3.1) with $\left|\frac{\partial u_{i}}{\partial z}\right|, i=1,2$, have the same order $h^{3} \gamma^{1 / 2} h^{2} \gamma^{1} h^{-2} \gamma^{-1} h^{-2}=h \gamma^{1 / 2}$. The other terms on $|U ; \Xi(h)|_{\gamma, 0}$ have the lower order $h \gamma^{3 / 2}$. For the field (5.30) all the terms of (3.1) with the factor $\min \{1, \gamma\}$ have the same order as the energy functional. This field also verifies the terms of (3.1) with $\left|U_{1}\right|$ and $\left|U_{2}\right|$.

\section{Korn's Inequality for the Isolated and for the Welded Rods}

\subsection{An Improved Weighted Korn Inequality for the Systems of Rods}

On one hand, it follows from (2.7) that

$$
\sum_{j: \Pi^{i, j}(h) \in \Pi^{i}(h)}\left|u ; \Pi^{i, j}(h)\right|_{i}^{2} \leq c \mathcal{E}_{\gamma}(u ; \Xi(h)) .
$$

On the other hand, we have according to (4.19)

$$
\min \{1, \gamma\}\left|u ; \Pi^{i}(h)\right|_{0}^{2} \leq c \mathcal{E}_{\gamma}(u ; \Xi(h)) .
$$


We introduce the norm

$$
\begin{aligned}
\left|u ; \Pi^{i}(h)\right|_{i, \bullet}:= & \sum_{j: \Pi^{i, j}(h) \in \Pi_{i}(h)} \int_{\Pi^{i, j}(h)}\left\{\left|\frac{\partial u_{1}}{\partial y_{1}}\right|^{2}+\left|\frac{\partial u_{2}}{\partial y_{2}}\right|^{2}+\left|\frac{\partial u_{3}}{\partial z}\right|^{2}\right. \\
& +\frac{h^{2}}{\rho_{h}^{2}}\left(\left|\frac{\partial u_{1}}{\partial y_{2}}\right|^{2}+\left|\frac{\partial u_{2}}{\partial y_{1}}\right|^{2}+\left|\frac{\partial u_{3}}{\partial y_{1}}\right|^{2}+\left|\frac{\partial u_{3}}{\partial y_{2}}\right|^{2}+\left|\frac{\partial u_{1}}{\partial z}\right|^{2}+\left|\frac{\partial u_{2}}{\partial z}\right|^{2}\right) \\
& \left.\left.+\frac{h^{2}}{\rho_{h}^{4}}\left|u_{3}\right|^{2}+\frac{1}{\rho_{h}^{2}}\left|u_{i}\right|^{2}+\beta_{1}(h, \gamma, y)\left|u_{3-i}\right|^{2}\right\} d y d z\right)^{1 / 2}
\end{aligned}
$$

and again obtain another Korn inequality for the systems $\Pi^{i}$.

Lemma 6.1 For $u \in H_{0}^{1}(\Xi(h))^{3}$ the following inequality holds

$$
\left|u ; \Pi^{i}(h)\right|_{i, \bullet}^{2} \leq c \mathcal{E}_{\gamma}(u ; \Xi(h)),
$$

where $c$ is a constant independent of $u$ and $h \in\left(0, h_{0}\right]$.

Proof Let $i=2$ (for $i=1$ we may give a similar proof). We put the origin of the coordinate system at the center of the cylinder $G_{1}(h)$. On the cell $S(h)$ we represent the vector $u$ in the form

$$
u(x)=u^{\perp}(x)+d(x) b
$$

where $b \in \mathbb{R}^{6}$ and $u^{\perp}$ satisfies the condition

$$
\int_{G_{2}(h)} d(x)^{\top} u^{\perp}(x) d x=0 .
$$

Note that in (6.6) we integrate over $G_{2}(h)$ in contrast to the integral in (5.4).

At first we estimate a norm for the second term in (6.5). We put $d b$ in (6.3) and obtain the inequality

$$
\begin{aligned}
\left|d b ; G_{2}(h)\right|_{i, \bullet}^{2} \leq & c h^{3}\left(h^{2} \kappa_{h}^{-2}\left(b_{3}^{2}+b_{4}^{2}+b_{5}^{2}\right)+h^{2} \kappa_{h}^{-4}\left(b_{3}^{2}+b_{4}^{2} y_{2}^{2}+b_{5}^{2} y_{1}^{2}\right)\right. \\
& \left.+\kappa_{h}^{-2}\left(b_{2}^{2}+b_{4}^{2} z^{2}+b_{6}^{2} y_{1}^{2}\right)+\mu_{1}\left(b_{1}^{2}+b_{5}^{2} z^{2}+b_{6}^{2} y_{2}^{2}\right)\right) \\
\leq & c h^{3}\left(h^{2} \kappa_{h}^{-2}\left(b_{3}^{2}+b_{4}^{2}+b_{5}^{2}\right)+h^{2} \kappa_{h}^{-4} b_{3}^{2}+\kappa_{h}^{-2} b_{2}^{2}+\mu_{1} b_{1}^{2}\right) .
\end{aligned}
$$

In order to estimate $b_{1}$ we multiply (6.5) by $e_{1}$ and integrate over $\Sigma_{2}(h)=$ $\partial G_{2}(h) \backslash \partial S(h)$ :

$$
\left|\Sigma_{2}(h)\right| b_{1}=\int_{\Sigma_{2}(h)}\left(u_{1}(x)-u_{1}^{\perp}(x)\right) d s_{x} .
$$

By the Cauchy-Bunyakowsky-Schwarz inequality we have

$$
\left|b_{1}\right|^{2} \leq \operatorname{ch}^{-2}\left(\mu_{1}^{-2} \int_{\Sigma_{2}(h)} \beta_{1}^{2} u_{1}(x)^{2} d s_{x}+\int_{\Sigma_{2}(h)} u_{1}^{\perp}(x)^{2} d s_{x}\right) .
$$

From (6.8) and (5.9) with $U=u_{1}^{\perp}$ and $U=\rho_{h}^{2} u_{1}$ it follows that 


$$
\begin{aligned}
\left|b_{1}\right|^{2} & \leq c h^{-3}\left(\mu_{1}^{-2} \int_{Q(h)} \beta_{1}^{2}\left(h^{2}\left|\nabla_{y} u_{1}\right|^{2}+\left|u_{1}\right|^{2}\right) d x+h^{2}\left\|\mid u_{1} ; Q(h)\right\|_{h}\right) \\
& \leq c h^{-3}\left(\mu_{1}^{-2}|u ; Q(h)|_{\gamma, \bullet}^{2}+\gamma^{-1} h^{2} \mathcal{E}_{\gamma}(u ; Q(h))\right) \\
& \leq c h^{-3} \mu_{1}^{-2}\left(|u ; Q(h)|_{\gamma, \bullet}^{2}+\mathcal{E}_{\gamma}(u ; Q(h))\right) .
\end{aligned}
$$

It is not necessary to apply the trace inequality for the estimate of the other terms $\left|b_{k}\right|$ of (6.7). Indeed, the required estimate follows immediately from (2.7). For instance, we multiply (6.5) by $e_{2}$, integrate over $\Sigma_{2}(h)$ and derive the estimate

$$
\begin{aligned}
\left|b_{2}\right|^{2} & \leq c h^{-3} \kappa_{h}^{2}\left(\int_{G_{2}(h)} \rho_{h}^{-2} u_{2}^{2}(x) d x+\int_{G_{2}(h)} \rho_{h}^{-2} u_{2}^{\perp}(x)^{2} d x\right) \\
& \leq c h^{-3} \kappa_{h}^{2}\left(\left|u ; G_{2}(h)\right|_{i}^{2}+\left\|u ; G_{2}(h)\right\|_{h}^{2}\right) \\
& \leq c h^{-3} \kappa_{h}^{2}\left(\left|u ; G_{2}(h)\right|_{i}^{2}+\mathcal{E}\left(u ; G_{2}(h)\right)\right) .
\end{aligned}
$$

In order to estimate $\left|b_{3}\right|$, we take the scalar product of (6.5) with $e_{3}$ and get

$$
\begin{aligned}
\left|b_{3}\right|^{2} & \leq c h^{-3} \kappa_{h}^{4} h^{-2}\left(h^{2} \int_{G_{2}(h)} \rho_{h}^{-4} u_{3}^{2}(x) d x+h^{2} \int_{G_{2}(h)} \rho_{h}^{-4} u_{3}^{\perp}(x)^{2} d x\right) \\
& \leq c h^{-5} \kappa_{h}^{2}\left(\left|u ; G_{2}(h)\right|_{i}^{2}+\|\| u ; G_{2}(h) \|_{h}^{2}\right) \\
& \leq c h^{-5} \kappa_{h}^{2}\left(\left|u ; G_{2}(h)\right|_{i}^{2}+\mathcal{E}\left(u ; G_{2}(h)\right)\right) .
\end{aligned}
$$

Similarly one obtains estimates for $\left|b_{3+j}\right|, j=1,2,3$, by taking scalar products of (6.5) with $x_{m} e_{n}$ and corresponding $n$ and $m$. Hence, from (6.7) it follows that

$$
\left|d b ; G_{2}(h)\right|_{i, \bullet}^{2} \leq c\left(\left|u ; G_{2}(h)\right|_{i}^{2}+\mathcal{E}\left(u ; G_{2}(h)\right)\right) .
$$

By definition of the norm $\left|\cdot ; G_{2}(h)\right|_{i}$ in (2.6) it holds that

$$
\left|u^{\perp} ; G_{2}(h)\right|_{i}^{2} \leq c \mid\left\|u^{\perp} ; G_{2}(h)\right\| \|_{h}^{2} \leq c \mathcal{E}\left(u ; G_{2}(h)\right) .
$$

Indeed, the second estimate in (6.11) follows from Theorem 2.3.3 [17]. We sum (6.11), (6.12) over all cells of the junction $\Xi(h)$ and arrive at the inequality

$$
\sum_{q}\left|u ; \Pi_{i}(h)\right|_{i, \bullet} \leq c\left(|u ; \Theta(h)|_{\gamma}^{2}+\left|u ; \Pi^{i}(h)\right|_{i}^{2}+\sum_{q} \mathcal{E}\left(u ; \Pi^{i}(h)\right)\right) .
$$

This estimate yields (6.4) by means of (2.7).

We combine (6.1), (6.2) and (6.4), introduce the norm (3.4) and arrive at the final Theorem 3.2 on the Korn inequality for the system of fibers. Note that due to the relation $h^{2} \beta_{1} \leq h^{2} \rho_{h}^{-2}$ we cannot improve the coefficient $h^{2} \rho_{h}^{-2}$ for the derivatives $\partial u_{i} / \partial z$ and $\partial z / \partial u_{i}$ with the help of the argument in the proof of Lemma 5.1.

\subsection{The Weight Distribution for the Rods is Optimal}

Let us verify that the estimate (3.5) is asymptotically sharp. We take a rod $\Pi^{i, j}(h) \in \Pi^{i}(h)$ with length $O(1)$. To simplify the notation, we suppose that the origin of the coordinates 
$(y, z)$ belongs to this rod. Next we construct some auxiliary vector fields using the cut-off functions

$X_{h}^{\Pi}\left(y_{i}\right)=\chi\left(h^{-\alpha}\left(y_{i}-y_{i}^{0}\right)\right)\left(1-\chi\left(h^{-\alpha}\left(y_{i}-y_{i}^{0}\right)\right)\right), \quad X_{h}^{\Theta}(x)=\chi\left(h^{-1} r_{0} \operatorname{dist}\left(x ; \Pi^{i, j}(h)\right)\right)$.

Here $y_{i}^{0}$ is the $y_{i}$-coordinate of the point $P^{0} \in \Pi^{i, j}(h) \cap \partial \Omega_{h}, \chi$ is the cut-off function defined in the proof of Theorem 4.1 and $0<\alpha<1$. We take $r_{0}$ so that $\operatorname{supp} X_{h}^{\Theta}(x) \cap$ $\Pi^{3-i, j}(h)=\emptyset$ and introduce the vector field

$$
U(x)=X_{h}^{\Theta}(x)\left(X_{h}^{\Pi}\left(y_{i}\right) e_{3-i}-y_{3-i} \frac{\partial}{\partial y_{i}} X_{h}^{\Pi}\left(y_{i}\right) e_{i}\right) .
$$

Note that $\mathcal{E}(U ; \Xi(h))=\mathcal{E}\left(U ; \Pi^{i, j}(h)\right)+\gamma \mathcal{E}(U ; \Theta(h))$ and

$$
\mathcal{E}\left(U ; \Pi^{i, j}(h)\right)=\left\|\varepsilon_{i i}(U) ; L_{2}\left(\Pi^{i, j}(h)\right)\right\|^{2} .
$$

According to the relation for the $3 \mathrm{~d}$-Lebesgue measure

$$
\operatorname{mes}_{3}\left(\operatorname{supp}\left\{X_{h}^{\Theta} \frac{\partial}{\partial y_{i}} X_{h}^{\Pi}\right\}\right)=O\left(h^{2+\alpha}\right)
$$

we have

$$
\mathcal{E}\left(U ; \Pi^{i, j}(h)\right)=O\left(h^{2} h^{2+\alpha} h^{-4 \alpha}\right) .
$$

The factors $h^{2}$ and $h^{-4 \alpha}$ appear in (6.15) due to the relation $\left|y_{3-i}\right|=O(h)$ for $y_{3-i} \in \Pi^{i, j}(h)$ and the estimate

$$
\left|\frac{\partial^{2}}{\partial y_{i}^{2}} X_{h}^{\Pi}\left(y_{i}\right)\right|=O\left(h^{-2 \alpha}\right) .
$$

By (6.14) and the estimate

$$
\left|\nabla X_{h}^{\Theta}(x)\right|=O\left(h^{-1}\right)
$$

we obtain

$$
\gamma \mathcal{E}(U ; \Theta(h))=O\left(\gamma h^{2+\alpha} h^{-2}\right)=O\left(\gamma h^{\alpha}\right) .
$$

Let us consider the case

$$
\gamma h^{-2} \leq h^{2} \rho_{h}^{-4}
$$

For $x \in \operatorname{supp} X_{h}^{\Theta}(x)$ we have

$$
c h^{\alpha} \leq \rho_{h}(x) \leq C h^{\alpha} .
$$

According to (6.17) and (6.18) the inequality $\gamma h^{\alpha} \leq h^{4-3 \alpha}$ holds. Therefore,

$$
\mathcal{E}(U ; \Xi(h)) \simeq \mathcal{E}\left(U ; \Pi^{i, j}(h)\right)=O\left(h^{4-3 \alpha}\right) .
$$

Due to (4.25) with $k=4$ the norm (3.4) has the same order $h^{4-3 \alpha}$.

If $h^{2} \rho_{h}^{-4}<\gamma h^{-2}<\rho_{h}^{-2}$, then by (6.17) and (6.18)

$$
\mathcal{E}(U ; \Xi(h)) \simeq \mathcal{E}(U ; \Theta(h))=O\left(\gamma h^{\alpha}\right)
$$

and due to (6.14) the norm (3.4) has the same order $\gamma h^{\alpha}$. 
Finally, in the case

$$
\rho_{h}^{-2} \leq \gamma h^{-2}
$$

we consider the vector field

$$
U(x)=\left(e_{1}+e_{2}+e_{3}\right) \chi\left(h^{-1} r_{0}\left|x-x_{0}\right|\right) .
$$

We take $r_{0}$ and $x_{0}$ such that $\operatorname{supp} \chi\left(h^{-1} r_{0}\left|x-x_{0}\right|\right)=\mathbb{B}_{h}$ belongs to $\Pi^{i, j}(h)$ and $\operatorname{dist}\left(\operatorname{supp} \chi\left(h^{-1} r_{0}\left|x-x_{0}\right|\right), \Gamma^{i, j}(h)\right)=O(h)$. Then all terms in (3.5) have order $h^{1}$.

\subsection{An Improved Weighted Korn Inequality for Plate with Welded Rods}

Let us confirm (2.10). We prove this inequality by repeating the scheme of the proofs of Lemma 4.1 and Lemma 6.1 with some simplifications (we do not use a trace inequality).

On the cell $S(h)$ we represent vector $u$ in the form of (6.5). From (5.11) it follows that

$$
h\left\|u^{\perp} ; Q(h)\right\|_{h}^{2} \leq c \mathcal{E}_{h}\left(u^{\perp} ; Q(h)\right) .
$$

In order to estimate $d(x) b$ we introduce the norm

$$
\begin{aligned}
|u ; \Theta(h)|_{\times, \bullet}:= & \left(\int _ { \Theta ( h ) } \left\{\sum _ { j = 1 } ^ { 2 } \left[h\left|\frac{\partial u_{j}}{\partial y_{1}}\right|^{2}+h\left|\frac{\partial u_{j}}{\partial y_{2}}\right|^{2}+\frac{h^{2}}{\rho_{h}^{2}}\left(\left|\frac{\partial u_{j}}{\partial z}\right|^{2}+\left|\frac{\partial u_{3}}{\partial y_{j}}\right|^{2}\right)\right.\right.\right. \\
& \left.\left.\left.+\frac{1}{\rho_{h}^{2}}\left|u_{j}\right|^{2}\right]+\left|\frac{\partial u_{3}}{\partial z}\right|^{2}+\frac{h^{2}}{\rho_{h}^{4}}\left|u_{3}\right|^{2}\right\} d y d z\right)^{1 / 2} .
\end{aligned}
$$

We put $d b$ in (6.20) and similarly to (6.7) obtain the inequality

$$
|u ; \Theta(h)|_{\times, \bullet} \leq c h^{3}\left(h^{2} \kappa_{h}^{-2}\left(b_{3}^{2}+b_{4}^{2}+b_{5}^{2}\right)+h^{2} \kappa_{h}^{-4} b_{3}^{2}+\kappa_{h}^{-2} b_{2}^{2}+\kappa_{h}^{-2} b_{1}^{2}\right)
$$

Estimates of the terms $\left|b_{k}\right|$ of (6.7) follow from (2.8). For instance, similarly as in (6.9), (6.10), we multiply (6.21) by $e_{1}$, integrate over $\Sigma_{2}(h)$ and derive the estimate

$$
\begin{aligned}
\left|b_{1}\right|^{2} & \leq c h^{-3} \kappa_{h}^{2}\left(\int_{G_{2}(h)} \rho_{h}^{-2} u_{1}^{2}(x) d x+\int_{G_{2}(h)} \rho_{h}^{-2} u_{1}^{\perp}(x)^{2} d x\right) \\
& \leq c h^{-3} \kappa_{h}^{2}\left(\left|u ; G_{2}(h)\right|_{\times, i}^{2}+\left\|u ; G_{2}(h)\right\| \|_{h}^{2}\right) \\
& \leq c h^{-3} \kappa_{h}^{2}\left(\left|u ; G_{2}(h)\right|_{\times, i}^{2}+\mathcal{E}\left(u ; G_{2}(h)\right)\right) .
\end{aligned}
$$

In the same way we obtain the estimates for $\left|b_{k}\right|, k>1$ by taking scalar products of (6.5) with $e_{j}, x_{m} e_{n}$ and corresponding $j, n, m$ and come to the inequality

$$
|d b ; \Theta(h)|_{\times, \bullet}^{2} \leq c\left(\left|u ; G_{2}(h)\right|_{i}^{2}+\mathcal{E}\left(u ; G_{2}(h)\right)\right) .
$$

We sum (6.22), (6.19) over all cells of the junction $\Xi(h)$ and arrive at the inequality according to the definition (2.11) of the norm $|u ; \Theta(h)|_{\times, 0}$ arrive at the (2.10). 


\section{References}

1. Akimova, E.A., Nazarov, S.A., Chechkin, G.A.: Asymptotics of a solution to a problem on deformation of an arbitrary local periodic thin plate. Trans. Mosc. Math. Soc. 65, 1-29 (2004)

2. Blanchard, D., Griso, G.: Decomposition of deformations of thin rods. Application to nonlinear elasticity. Anal. Appl. 7, 21-71 (2009)

3. Blanchard, D., Gaudiello, A., Griso, G.: Junction of a periodic family of elastic rods with a thin plate. II. J. Math. Pures Appl. 88, 149-190 (2007)

4. Blanchard, D., Gaudiello, A., Griso, G.: Junction of a periodic family of elastic rods with a $3 \mathrm{~d}$ plate. I. J. Math. Pures Appl. 88(1), 1-33 (2007)

5. Cioranescu, D., Oleinik, O.A., Tronel, G.: Korn's inequalities for frame type structures and junctions with sharp estimates for the constants. Asymptot. Anal. 8, 1-14 (1994)

6. Duvaut, G., Lions, J.L.: Les Inéquations en Mécanique et en Physique. Dunod, Paris (1972)

7. Friedrichs, K.O.: On the boundary value problems of the theory of elasticity and Korn's inequality. Ann. Math. 48, 447-471 (1947)

8. Griso, G.: Asymptotic behavior of structures made of plates. Anal. Appl. 3(4), 325-356 (2005)

9. Griso, G.: Decompositions of displacements of thin structures. J. Math. Pures Appl. 89, 199-223 (2008)

10. Griso, G.: Asymptotic behavior of structures made of curved rods. Anal. Appl. 6(1), 11-22 (2008)

11. Izotova, O.V., Nazarov, S.A., Sweers, G.H.: Weighted Korn inequalities for thin-walled elastic structures. C. R., Méc. 334, 707-712 (2006)

12. Izotova, O.V., Nazarov, S.A., Sweers, G.H.: Asymptotically sharp weighted Korn inequalities for thinwalled elastic constructions. J. Math. Sci. 150, 1807-1855 (2008)

13. Kondrat'ev, V.A., Olejnik, O.A.: Boundary value problems for the system of elasticity theory in unbounded domains. Korn's inequalities. Russ. Math. Surv. 43, 65-119 (1988)

14. Kondrat'ev, V.A., Olejnik, O.A.: On Korn's inequalities. C. R. Math. 308, 483-487 (1989)

15. Korn, A.: Die Eigenschwingungen eines elastischen Körpers mit ruhender Oberfläche. Acad. Wiss., Munich, Math.-Phys. Kl. 36, 351-401 (1906)

16. Korn, A.: Solution générale du problème d'équilibre dans la théorie de l'élasticité dans le cas où les efforts sont donnés à la surface. Ann. Fac. Sci. Toulouse 10, 165-269 (1908)

17. Lekhnitskii, S.G.: Theory of Elasticity of an Anisotropic Elastic Body. Holden-Day, San Francisco (1963). Translated by P. Fern. Edited by Julius J. Brandstatter

18. Nazarov, S.A.: Korn inequalities that are asymptotically exact for thin domains. Vestn. St.-Peterbg. Univ., Ser. 1 Mat. Meh. Astron. 2, 19-24, 113-114 (1992) (Russian); translation in Vestn. St. Petersburg Univ. Math. 25(2), 18-22 (1992)

19. Nazarov, S.A.: Korn's inequalities for junctions of spatial bodies and thin rods. Math. Methods Appl. Sci. 20, 219-243 (1997)

20. Nazarov, S.A.: Justification of the asymptotic theory of thin rods. Integral and pointwise estimates. Problems of mathematical physics and function theory. J. Math. Sci. (N.Y.) 97, 4245-4279 (1999)

21. Nazarov, S.A.: Asymptotic Theory of Thin Plates and Rods, vol. 1. Dimension Reduction and Integral Estimates. Nauchnaya Kniga, Novosibirsk (2002) [in Russian]

22. Nazarov, S.A.: Weighted anisotropic Korn's inequality for a junction of a plate and rods. Mat. Sb. 195, 97-126 (2004) (Russian); translation in Sb. Math. 195, 553-583 (2004)

23. Nazarov, S.A.: Korn's inequalities for elastic joints of massive bodies, thin plates, and rods. Usp. Mat. Nauk 63, 37-110 (2008) (Russian); translation in Russ. Math. Surv. 63, 35-107 (2008)

24. Nazarov, S.A., Slutskii, A.S.: Korn's inequality for an arbitrary system of thin distorted rods. Sib. Math. J. 43, 1319-1331 (2002)

25. Nazarov, S.A., Slutskii, A.S., Sweers, G.: Homogenization in a thin plate reinforced with periodic families of stiff fibers. Sb. Math. (2010, to appear)

26. Nečas, J.: Les Méthodes Directes en Théorie des Équations Elliptiques. Masson, Paris (1967)

27. Payne, L.E., Weinberger, H.F.: On Korn's inequality. Arch. Ration. Mech. Anal. 8(2), 89-98 (1961)

28. Shoikhet, B.A.: On asymptotically exact equations of thin plates of complex structure. J. Appl. Math. Mech. 37(1973), 867-877 (1974); translated from Prikl. Mat. Meh. 37, 914-924 (1973) 